\title{
OPEN Reciprocal regulation of endothelial-mesenchymal transition by MAPK7 and EZH2 in intimal hyperplasia and coronary artery disease
}

Byambasuren Vanchin ${ }^{1,2,5}$, Marloes Sol ${ }^{1,5}$, Rutger A. F. Gjaltema ${ }^{1}$, Marja Brinker ${ }^{1}$, Bianca Kiers ${ }^{3}$, Alexandre C. Pereira ${ }^{3}$, Martin C. Harmsen ${ }^{1}$, Jan-Renier A. J. Moonen ${ }^{1,4}$ \& Guido Krenning ${ }^{1 \bowtie}$

Endothelial-mesenchymal transition (EndMT) is a form of endothelial dysfunction wherein endothelial cells acquire a mesenchymal phenotype and lose endothelial functions, which contributes to the pathogenesis of intimal hyperplasia and atherosclerosis. The mitogen activated protein kinase 7 (MAPK7) inhibits EndMT and decreases the expression of the histone methyltransferase Enhancerof-Zeste homologue 2 (EZH2), thereby maintaining endothelial quiescence. EZH2 is the catalytic subunit of the Polycomb Repressive Complex 2 that methylates lysine 27 on histone 3 (H3K27me3). It is elusive how the crosstalk between MAPK7 and EZH2 is regulated in the endothelium and if the balance between MAPK7 and EZH2 is disturbed in vascular disease. In human coronary artery disease, we assessed the expression levels of MAPK7 and EZH2 and found that with increasing intima/ media thickness ratio, MAPK7 expression decreased, whereas EZH2 expression increased. In vitro, MAPK7 activation decreased EZH2 expression, whereas endothelial cells deficient of EZH2 had increased MAPK7 activity. MAPK7 activation results in increased expression of microRNA (miR)101 , a repressor of EZH2. This loss of EZH2 in turn results in the increased expression of the miR-200 family, culminating in decreased expression of the dual-specificity phosphatases 1 and 6 who may repress MAPK7 activity. Transfection of endothelial cells with miR-200 family members decreased the endothelial sensitivity to TGF $\beta 1$-induced EndMT. In endothelial cells there is reciprocity between MAPK7 signaling and EZH2 expression and disturbances in this reciprocal signaling associate with the induction of EndMT and severity of human coronary artery disease.

Neointimal hyperplasia is characterized by an increasing amount of fibroproliferative cells and extracellular matrix in the neointimal lesion, resulting in vascular lumen narrowing - characterized by an increasing intima/ media thickness ratio (IMT) in histopathology—and eventually obstruction of the vessel. Endothelial cells play a pivotal role in the formation of neointimal lesions by the acquisition of a fibro-proliferative phenotype through endothelial-to-mesenchymal transition $(\text { EndMT })^{1-5}$. EndMT is characterized by a change from an endothelial phenotype into a phenotype comprising of mesenchymal-like properties, in which the expression of endothelial cells markers, such as eNOS, PECAM-1 and VE-cadherin is lost, and the expression of mesenchymal genes,

${ }^{1}$ Laboratory for Cardiovascular Regenerative Medicine, Department of Pathology and Medical Biology, University Medical Center Groningen, University of Groningen, Hanzeplein 1 (EA11), 9713GZ Groningen, The Netherlands. ${ }^{2}$ Department of Cardiology, School of Medicine, Mongolian National University of Medical Sciences, Jamyan St 3, Ulaanbaatar 14210, Mongolia. ${ }^{3}$ Laboratory of Genetics and Molecular Cardiology (LIM13), Heart Institute (InCor), University of São Paulo, Avenida Dr. Eneas C. Aguiar 44, São Paulo, SP 05403-000, Brazil. 'Department of Pediatric Cardiology, Center for Congenital Heart Diseases, Beatrix Children's Hospital, University Medical Center Groningen, University of Groningen, Hanzeplein 1 (CA40), 9713GZ Groningen, The Netherlands. ${ }^{5}$ These authors contributed equally: Byambasuren Vanchin and Marloes Sol. ${ }^{\varpi}$ email: g.krenning@ umcg.nl 
including SM22a, aSMA and vimentin, is gained. Moreover, EndMT-derived fibroproliferative cells secrete extracellular matrix components, which might contribute to the buildup of the neointima ${ }^{6}$.

EndMT was originally identified during embryogenesis, where it plays a pivotal role in cardiac valve, septum and endocardial cushion formation ${ }^{7}$. In adults, EndMT contributes to fibroproliferative diseases, including atherosclerosis $^{1-5}$, cerebral cavernous malformation ${ }^{8}$, pulmonary fibrosis ${ }^{9}$, kidney fibrosis ${ }^{10}$ and cardiac fibrosis ${ }^{11}$.

Uniform laminar shear stress (LSS) conveys atheroprotective effects to the endothelium, while endothelial cells exposed to disturbed or low oscillatory shear stress are prone to EndMT ${ }^{12,13}$. Uniform LSS activates the mitogen-activated protein kinase 7 (MAPK7) —also known as extracellular signal-related kinase 5 (Erk5) and big-mitogen kinase-1 (BMK-1) - which suppresses EndMT ${ }^{5,14,15}$. Concurrently, the loss of MAPK7 signaling facilitates EndMT ${ }^{5,16}$. Currently, it is elusive how MAPK7 activity is regulated in fibroproliferative disease.

The histone methyltransferase Enhancer of Zeste Homolog 2 (EZH2), which is the catalytic subunit of the Polycomb Repressive Complex 2, plays a pivotal role in endothelial dysfunction ${ }^{17-19}$. EZH2 is responsible for the trimethylation of lysine 27 on histone 3, which silences gene expression and is elevated in endothelial cells in atherosclerotic lesions ${ }^{20}$. Serendipically, we uncovered that uniform LSS reduces the expression of EZH2, whereas the RNAi-mediated repression of EZH2 reciprocally activates MAPK7 signaling in endothelial cells even in the absence of $\mathrm{LSS}^{18}$. Currently, it is elusive how the crosstalk between MAPK7 and EZH2 is regulated in the endothelium and whether the balance between MAPK7 and EZH2 is disturbed during intimal hyperplasia and coronary artery disease.

Here, we report that in the endothelium there is reciprocity between MAPK7 and EZH2 in the regulation of EndMT and in human coronary artery disease. In endothelial cells exposed to uniform LSS, the activation of MAPK7 increases the expression of microRNA (miR)-101, which in turn suppresses the translation of the EZH2 gene, resulting in reduced protein expression. Reciprocally, the reduced expression of EZH2 results in the decreased expression of the Dual Specificity Phosphatase (DUSP)-1 and DUSP-6 - the phosphatases responsible for the inactivation of MAPK $7^{21}$ - which facilitates the activation of MAPK7. Disturbance in the reciprocity between MAPK7 and EZH2 result in the induction of EndMT and associate to the degree of human coronary artery disease.

\section{Results}

Reciprocity between MAPK7 and EZH2 in human coronary artery disease. Human coronary artery samples were stratified into three groups based on their intima/media thickness ratio (IMT), ranging from IMT $<1 \mu \mathrm{m}_{\text {intima }} \cdot \mu \mathrm{m}_{\text {lumen }}{ }^{-1}$ to IMT $>3 \mu \mathrm{m}_{\text {intima }} \cdot \mu \mathrm{m}_{\text {lumen }}{ }^{-1}$; Fig. $\left.1 \mathrm{a}-\mathrm{d}\right)$. Mean age, and the hypertension, diabetes and smoking status did not differ between groups. Coronary artery disease is characterized by a progressively increasing intima-media thickness ratio (Fig. 1a-d), which coincides with the progressive narrowing of the coronary artery lumen (Fig. 1e). Coronary artery MAPK7 expression decreases with an increasing coronary artery intima-media thickness ratio $\left(\mathrm{r}^{2}=0.2517, \mathrm{p}=0.04\right.$; Fig. 1f,g). In contrast, EZH2 expression is elevated in coronary artery disease (Fig. $1 \mathrm{~h}, \mathrm{i}$ ) and an increasing coronary artery intima-media thickness ratio associates with increased EZH2 expression $\left(\mathrm{r}^{2}=0.4417, \mathrm{p}=0.004\right.$, Fig. 1i), suggesting reciprocity between MAPK7 and $\mathrm{EZH} 2$ in human coronary artery disease.

Reciprocal signaling between MAPK7 and EZH2 in endothelial cells. We recently uncovered that disturbed fluid shear stress (FSS) contributes to intima hyperplasia by the induction of endothelial-mesenchymal transition $(\mathrm{EndMT})^{5}$, partially mediated by EZH2 ${ }^{18}$. Atheroprotective uniform LSS decreases EZH2 expression at both the gene $(2.2$-fold, $\mathrm{p}<0.001$; Fig. $2 \mathrm{a})$ and protein $(1.9$-fold, $\mathrm{p}=0.028$; Fig. 2b; Suppl. Fig. 1$)$ level. Uniform laminar shear stress does not change the expression of MAPK7, neither on transcript (Fig. 2c) nor on protein level (Suppl. Fig. 1), however, FSS increases the activity of MAPK7 as indicated by the increased phosphorylation (3.5-fold, $\mathrm{p}=0.036$, Fig. 2d; Suppl. Fig. 1). Knockdown of EZH2 did not significantly alter MAPK7 transcript expression, whereas MAPK7 activity is increased upon EZH2 reduction (1.9-fold, $\mathrm{p}=0.049$; Fig. 2d). Moreover, protein expression levels of EZH2 associate with MAPK7 activation $\left(r^{2}=0.7723, p=0.021\right.$; Fig. 2e) proving evidence of the reciprocity between EZH2 expression levels and MAPK7 activity.

MAPK7 decreases EZH2 through miRNA-101. As MAPK7 decreases EZH2 post-transcriptionally ${ }^{18}$, we investigated whether miRNA-101-a known translational repressor of EZH2 in endothelial cells ${ }^{22}$ - is regulated by MAPK7 signaling. FSS increased the expression of miR-101 in a MAPK7-dependent manner (2.8-fold, $\mathrm{p}<0.001$; Fig. 3a). In luciferase reporter assays, miR-101 binds to the 3'UTR of EZH2, reducing the luminescence signal (1.9-fold, $\mathrm{p}<0.001$; Fig. 3b). In endothelial cells, ectopic expression of miR-101 decreases EZH2 expression at both the gene (2.6-fold, $p=0.002$; Fig. 3c) and protein (2.9-fold, $p=0.008$; Fig. 3d; Suppl. Fig. 2) level, whereas miRNA-101 did not significantly alter MAPK7 gene expression (Fig. 3e) or MAPK7 protein expression level (Fig. 3f; Suppl. Fig. 2). In human coronary artery disease, $m i R-101$ expression is decreased (p $<0.01$, Fig. 3g) and increased disease severity, i.e. increasing IMT, associates with a progressive decrease in $m i R-101\left(\mathrm{r}^{2}=0.4452\right.$, $\mathrm{p}=0.003$, Fig. $3 \mathrm{~h})$. Moreover, the expression level of miR-101 associates with MAPK7 $\left(\mathrm{r}^{2}=0.4262, \mathrm{p}=0.005\right.$; Fig. $3 \mathrm{i})$ and tends to associate to EZH2 $\left(\mathrm{r}^{2}=0.2304, \mathrm{p}=0.051\right.$; Fig. $\left.3 \mathrm{j}\right)$ in coronary artery disease, where a negative association between MAPK7 and EZH2 expression $\left(r^{2}=0.2568, p=0.038\right.$; Fig. $\left.3 \mathrm{k}\right)$ is present. Collectively, these data suggest that in coronary artery disease, the reciprocity between MAPK7 activity and EZH2 expression is regulated by miR-101.

EZH2 regulates DUSP-1 and DUSP-6 expression through miR200a-c. EZH2 expression determines the level of MAPK7 activity. However, EZH2 is a transcriptional repressor that cannot directly regulate the activity of a kinase. MAPK7 activity is regulated by the Dual Specificity Phosphatases (DUSP)-1 and DUSP- $6^{21}$, 
a

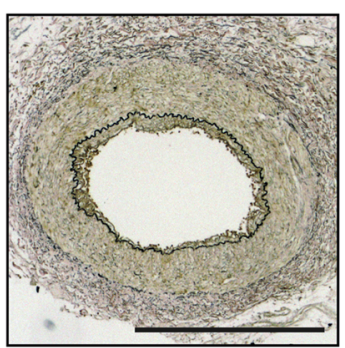

f

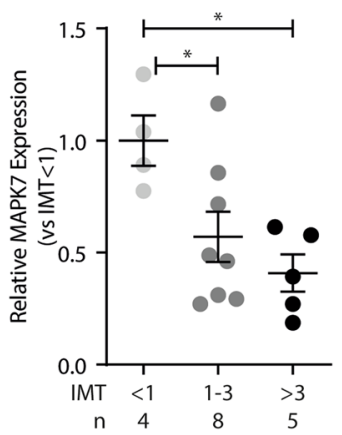

b

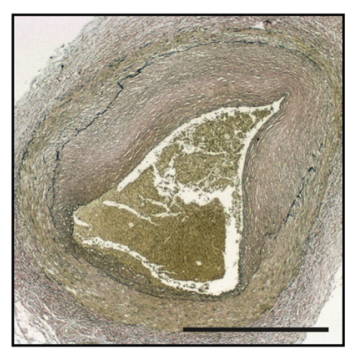

g

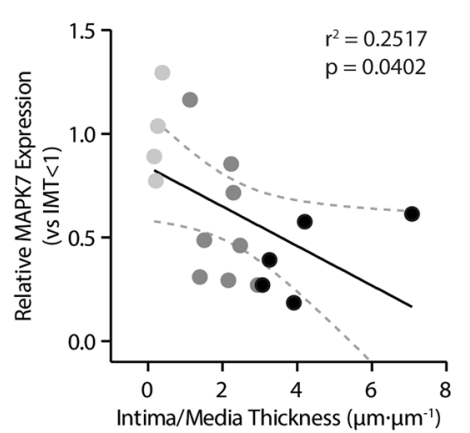

d

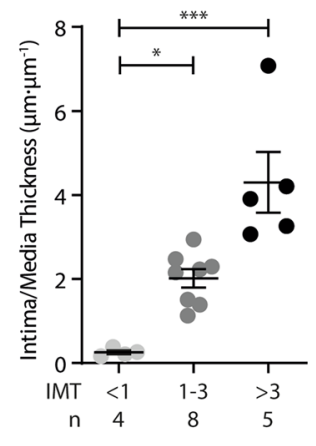

e

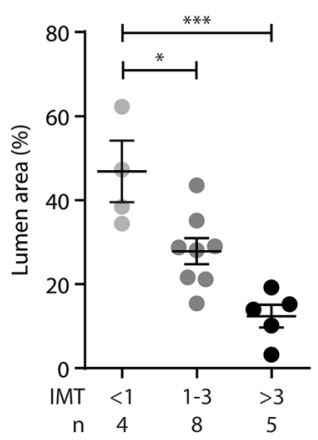

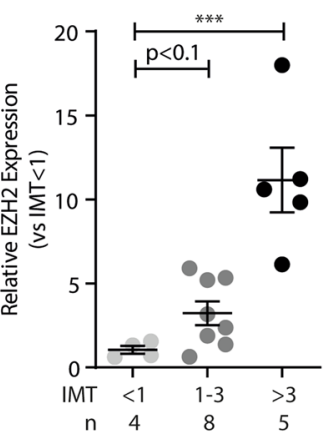

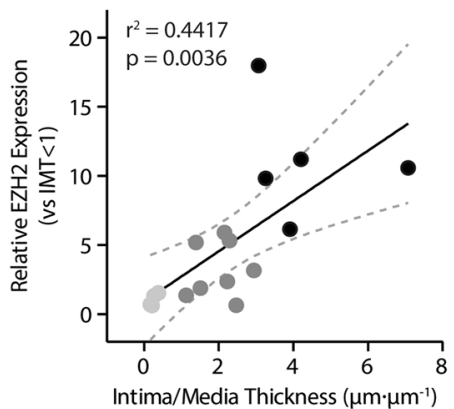

Figure 1. Reciprocity between MAPK7 and EZH2 in human coronary artery disease. (a-c) Representative pictures of Verhoeff-stained human coronary artery samples $(n=4-8)$ with increasing IMT, IMT $<1$ (a) IMT 1-3 (b) and IMT $>3$ (c). Intima-media thickness was measured $\left(\mu \mathrm{m}_{\text {intima }} / \mu \mathrm{m}_{\text {media }}\right)$ and samples stratified into three groups based on their intima-media thickness (d). An increasing IMT coincides with a progressively decreasing lumen area of the coronary artery (e), suggestive of progressive stenosis. MAPK7 expression levels were determined by qPCR and normalized to IMT < 1 (f). MAPK7 decreases with increasing IMT (g). EZH2 expression levels were determined by qPCR and normalized to IMT $<1$ (h). EZH2 expression increased with increasing IMT (i). Data is expressed as mean \pm S.D. of all individual observations. Statistical analysis was performed by ANOVA followed by Bonferroni post hoc tests. Correlations were performed using Pearson correlation. ${ }^{\star} \mathrm{p}<0.05,{ }^{\star * *} \mathrm{p}<0.001$
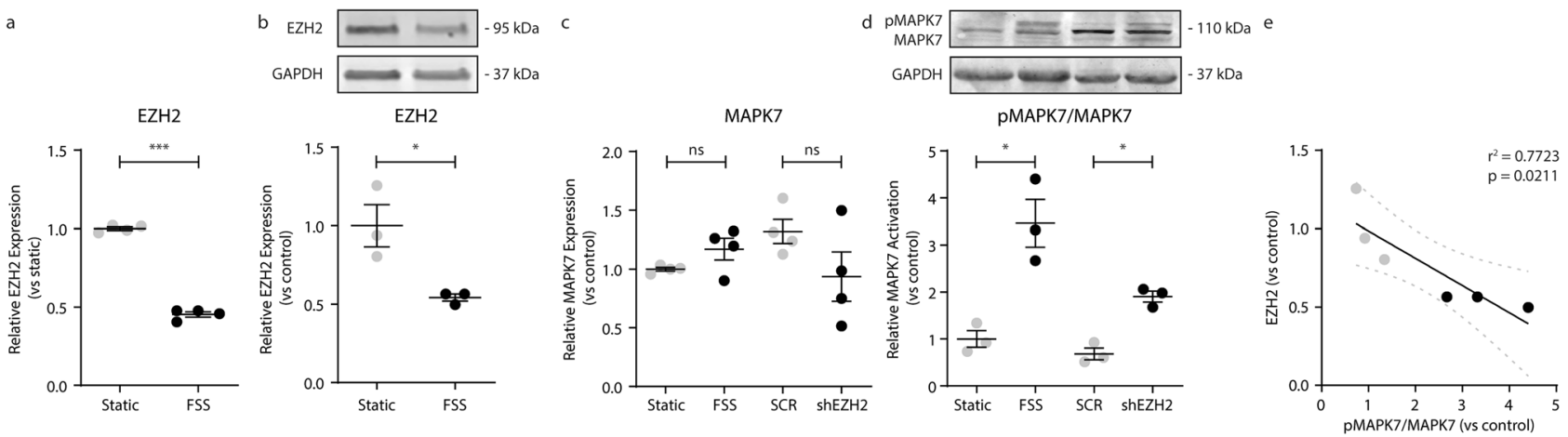

Figure 2. Reciprocal signaling between MAPK7 and EZH2 in endothelial cells. EZH2 expression levels were determined by qPCR in HUVEC exposed to FSS $\left(20\right.$ dyne $\left./ \mathrm{cm}^{2}\right)$ compared to static controls (a). EZH2 protein levels were determined by western blot in HUVEC exposed to FSS and compared to static control (b). MAPK7 expression levels were determined by qPCR in HUVEC exposed to FSS, and HUVEC that are deficient in EZH2 (shEZH2) (c). MAPK7 activation (pMAPK7) levels were determined by immunoblotting and normalized to total MAPK7 protein levels (d). Protein expression of EZH2 and MAPK7 activation were associated in endothelial cells (e). Data is expressed as mean \pm S.D. of all individual observations. Gene and protein expression data were obtained from 4 and 3 independent experiments, respectively. Comparisons between 2 groups were performed by Student t-tests and data from multiple groups were analyzed by ANOVA followed by Bonferroni post hoc tests. Correlations were performed using Pearson correlation. ${ }^{\star * *} \mathrm{p}<0.001$. 

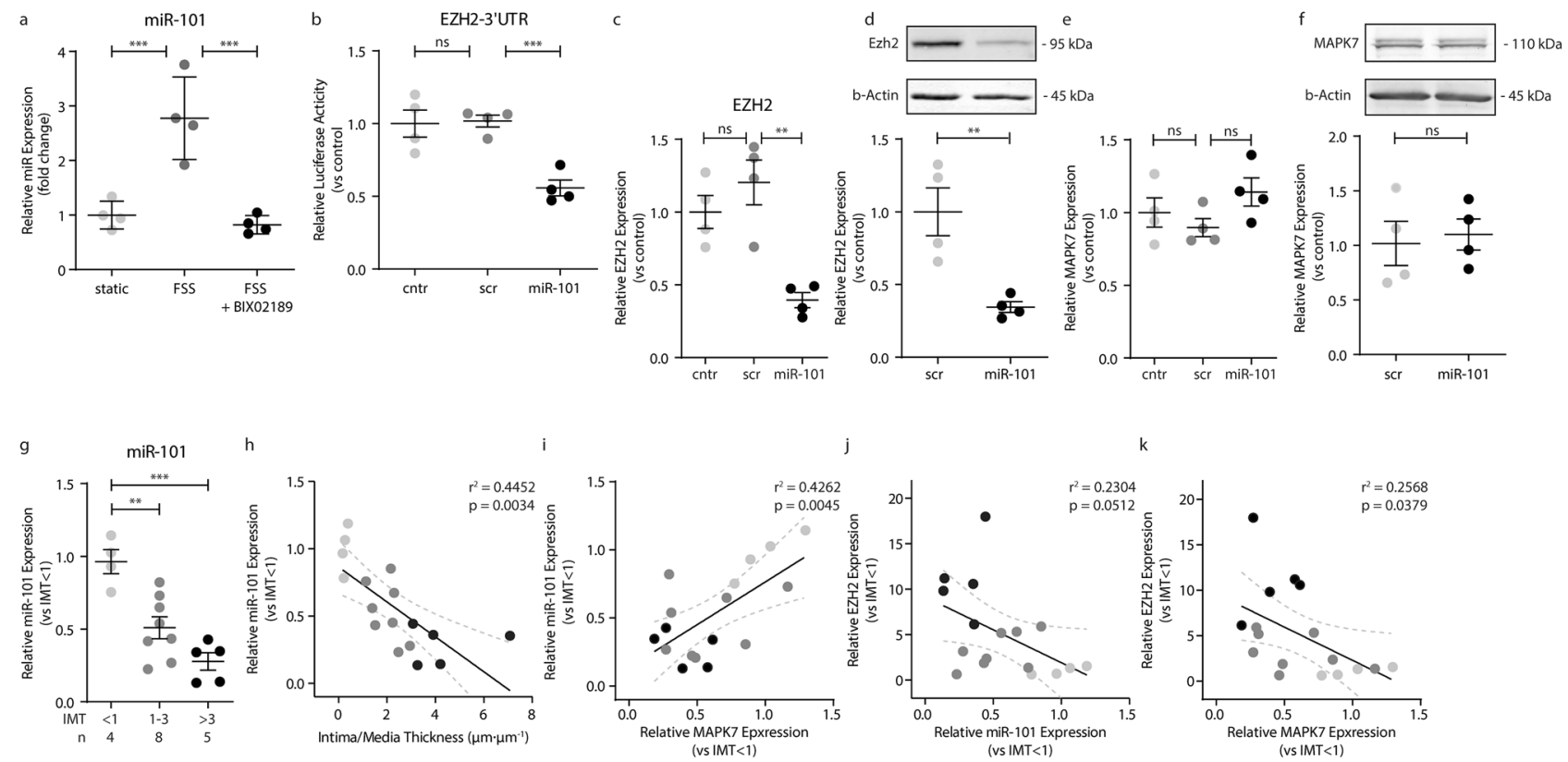

Figure 3. MAPK7 decreases EZH2 through miRNA-101. MiR-101 expression levels were determined by qPCR in HUVEC exposed to FSS $\left(20\right.$ dyne $\left./ \mathrm{cm}^{2}\right)$ with or without the MAPK7 inhibitor BIX02189 $(10 \mu \mathrm{M})$ and normalized to the level of static controls (a). Luciferase reporter binding assays were performed for the 3'UTR of EZH2 in COS7 cells with ectopic expression of miR-101 or scrambled control sequences (scr). Luciferase activity was normalized to non-transfected cells (b). EZH2 and MAPK7 expression levels were determined by qPCR in HUVEC with ectopic expression of miR-101 or SCR and normalized to control (c,e). EZH2 and MAPK7 protein levels were determined by western blot in HUVEC with ectopic expression of miR-101 or scrambled control sequences $(\mathbf{d}, \mathbf{f})$. MiR-101 expression levels were determined by qPCR and normalized to IMT $<1(\mathbf{g})$. MiR-101 decreases with increasing IMT (h), and associates with MAPK7 (i), and tends to associate with EZH2 (j) expression levels. In coronary artery disease, MAPK7 expression negatively correlates to EZH2 expression (k). Data is expressed as mean \pm S.D. of all individual observations. In vitro experimental data was derived from 4 independent experiments, whereas human in vivo data was derived from $n=4-8$ samples per group. Comparisons between 2 groups were performed by Student t-tests and data from multiple groups were analyzed by ANOVA followed by Bonferroni post hoc tests. Correlations were performed using Pearson correlation. ${ }^{*} \mathrm{p}<0.05,{ }^{* *} \mathrm{p}<0.01,{ }^{* *} \mathrm{p}<0.001$.

yet a reduction in EZH2 expression is associated with a decreased expression of DUSP- 1 and DUSP- $6^{23,24}$. Therefore, we investigated alternative mechanisms that might decrease DUSP expression upon the reduction of EZH2. In silico analysis, using Targetscan.org ${ }^{25}$ to identify microRNAs that putatively target DUSP-1 and DUSP-6 was followed by cross-referencing for EZH2 or $\mathrm{H} 3 \mathrm{~K} 27 \mathrm{Me} 3$ interactions in endothelial cells and mesenchymal cells using the genome browser (ENCODE Histone Modifications track set, genomebrowser.org for HUVEC, human microvascular endothelial cells (HMEC), normal human dermal fibroblasts (NHDF) and normal human lung fibroblasts (NHLF)) and putatively identifies the microRNA-200 family (miR-200a, miR-200b, miR-200c, miR141 and miR-429) as regulators of DUSP-1 and DUSP-6 with different ChIP signal between endothelial cells (low signal) and mesenchymal cells (high signal). Therefore, we investigated if the expression of the miR-200b/a/429 cluster on chromosome 1 and the miR-200c/141 cluster on chromosome 12 are under control of EZH2. Uniform LSS increased the expression of all microRNAs of the miR-200b/a/429 and miR-200c/141 clusters (Fig. 4a-e). Moreover, knockdown of EZH2 similarly increased the expression of microRNAs in these clusters (Fig. 4a-e).

The EZH2-induced trimethylation of lysine 27 on histone $3(\mathrm{H} 3 \mathrm{~K} 27 \mathrm{Me} 3)$ is present in the promoter regions of the miR-200b/a/429 and miR-200c/141 clusters (Fig. 4f,i). Knockdown of EZH2 in endothelial cells reduced the level of $\mathrm{H} 3 \mathrm{~K} 27 \mathrm{Me} 3$ at these gene regions (Fig. 4f,i), and the loss of this repressive histone mark coincided with increased expression of miR-200a-c, miR-141 and miR-429. In endothelial cells with constitutively active MAPK7 signaling (MEK5D), the abundance of $\mathrm{H} 3 \mathrm{~K} 27 \mathrm{Me} 3$ is decreased at the promoter regions of miR-200b/a/429 (1.6-fold, $p=0.034$; Fig. 4g,h) and miR-200c/141 (1.9-fold, $p=0.035$; Fig. 4j,k), implying that MAPK7 activity increases the expression of miR-200 family members through a decrease in EZH2-mediated gene silencing.

In luciferase reporter assays, all miR-200 family members were able to bind to the 3'UTR of DUSP-1 (Fig. 5a), but only miR-200a and miR-141 were able to bind the 3'UTR of DUSP-6 (Fig. 5c). Corroboratively, exogenous expression of all miR-200 family members in endothelial cells decreased DUSP-1 expression (Fig. 5b), whereas only miR-200a and miR-141 decreased the expression of DUSP-6 (Fig. 5d). Collectively, these data imply that the activation of MAPK7 by uniform LSS decreases the expression of DUSP-1 and DUSP-6 expression via the EZH2-dependent regulation of $\mathrm{miR}-200 \mathrm{~b} / \mathrm{a} / 429$ and $\mathrm{miR}-200 \mathrm{c} / 141$ expression. 

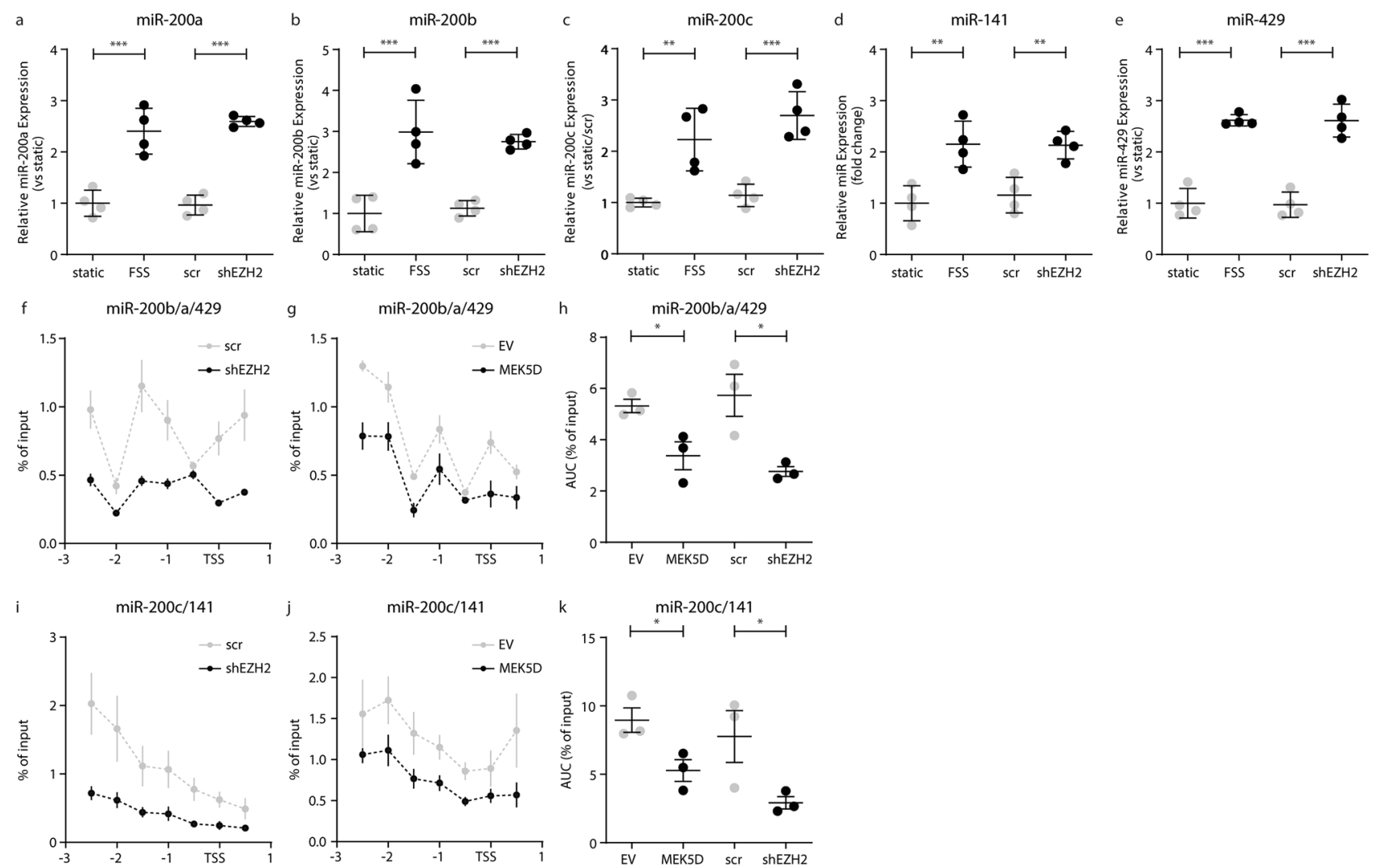

Figure 4. EZH2 regulates DUSP-1 and DUSP-6 expression through miR200a-c. MiR-200a (a), miR-200b (b), $m i R-200 c$ (c), $m i R-141$ (d), and miR-429 (e) expression levels were determined by qPCR in HUVEC exposed to FSS $\left(20\right.$ dyne $\left./ \mathrm{cm}^{2}\right)$, transduced HUVEC with scr or shEZH2 and normalized to static control cells. H3K27me3 enrichment around the transcription start site (TSS) of the miR-200b/a/429 cluster was determined using ChIP in shEZH2-HUVEC and scr-HUVEC (f) and MEK5D-HUVEC and EV-HUVEC (g). H3K27me3 enrichment is shown as area under the curve (AUC) compared to input samples (h). H3K27me3 enrichment at the around the TSS of miR-200c/141 cluster was determined using ChIP in scr-HUVEC and shEZH2 (i) and in MEK5DHUVEC and EV-HUVEC (j). H3K27me3 enrichment is shown as AUC compared to input samples (k). Data is expressed as mean \pm S.D. of all individual observations. Gene expression data were obtained from 4 independent experiments. Data from ChIP experiments were obtained from three independent experiments. All data was analyzed by ANOVA followed by Bonferroni post hoc tests. ${ }^{\star} \mathrm{P}<0.05,{ }^{*} \mathrm{P}<0.01,{ }^{\star *} \mathrm{P}<0.001$.

Pharmacological inhibition of DUSP activity does not alter MAPK7 activity and EZH2 expression. We investigated if the pharmacological inhibition of DUSP-1 and DUSP-6 activity in endothelial cells would activate MAPK7 signaling and decrease the expression of EZH2. BCI-treated endothelial cells modestly increased MAPK7 phosphorylation (1.7-fold; Fig. 6a; Suppl. Fig. 3), albeit not statistically significant $(p=0.202)$, and did not affect EZH2 expression (Fig. 6b; Suppl. Fig. 3). The addition of Simvastatin - a known activator of MAPK7 signaling ${ }^{15}$ - did increase increased MAPK7 activation (5.8-fold, p $<0.001$; Fig. 6a; Suppl. Fig. 3) and decreased EZH2 protein expression (3.0-fold, $\mathrm{p}<0.001$; Fig. $6 \mathrm{~b}$; Suppl. Fig. 3). The addition of BCI to simvastatin-treated endothelial cells did not increase the levels of MAPK7 activation nor decrease the protein expression of EZH2 further. Rather, the addition of BCI reduced the simvastatin-induced activity of MAPK by $27 \%$ ( $p=0.033$; Fig. 6a; Suppl. Fig. 3).

In human coronary artery disease, DUSP-1 expression is increased in advanced lesions (IMT $>3, \mathrm{p}<0.001$, Fig. 6c) and increasing IMT associates with increased DUSP-1 expression $\left(\mathrm{r}^{2}=0.2767, \mathrm{p}=0.0301\right.$; Fig. $\left.6 \mathrm{~d}\right)$. Moreover, the increase in DUSP-1 expression associates with increased $E Z H 2$ expression in coronary artery disease $\left(\mathrm{r}^{2}=0.4541, \mathrm{p}=0.0030\right.$; Fig. 6e $)$ and the increase in DUSP-1 expression tends to associate with decreased MAPK7 expression $\left(\mathrm{r}^{2}=0.1686, \mathrm{p}=0.1016\right.$; Fig. $\left.6 \mathrm{f}\right)$, although not significantly. Also, DUSP-6 seems to be increased in coronary artery disease ( $<<0.1$, Fig. $6 \mathrm{~g})$, albeit not significantly. The apparent increase in DUSP-6 expression does not significantly associate with increasing IMT $\left(\mathrm{r}^{2}=0.0681, \mathrm{p}=0.3116\right.$; Fig. $\left.6 \mathrm{~h}\right)$, nor do the expression levels of DUSP-6 significantly associate with EZH2 $\left(\mathrm{r}^{2}=0.0734, \mathrm{p}=0.2929\right.$; Fig. $\left.6 \mathrm{i}\right)$ and MAPK7 expression $\left(\mathrm{r}^{2}=0.0163\right.$, $\mathrm{p}=0.6251$; Fig. $6 \mathrm{j})$ in the coronary artery tissue.

Ectopic expression of miRNA-101, miRNA-141 and miRNA-200a inhibits endothelial dysfunction and EndMT. As coronary artery disease is associated with EndMT ${ }^{1,26}$, we investigated if the ectopic expression of miR-101 or miR-200 family members could preclude EndMT. Endothelial cells transfected with 


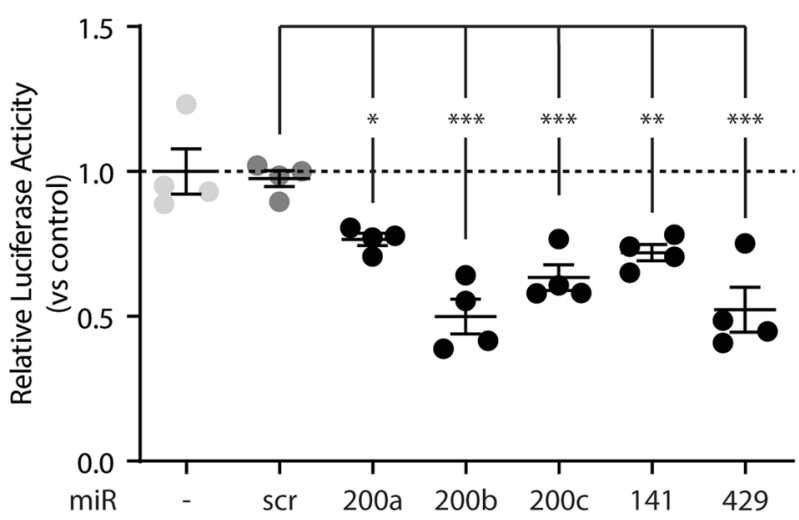

C

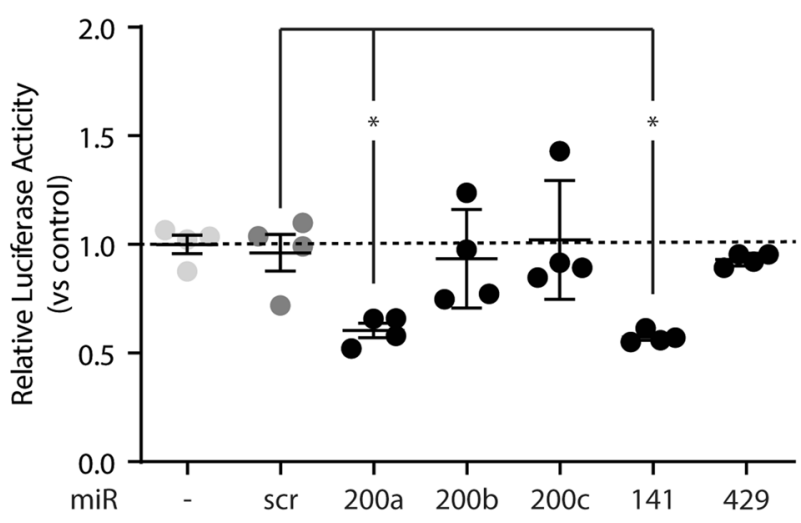

b

DUSP1

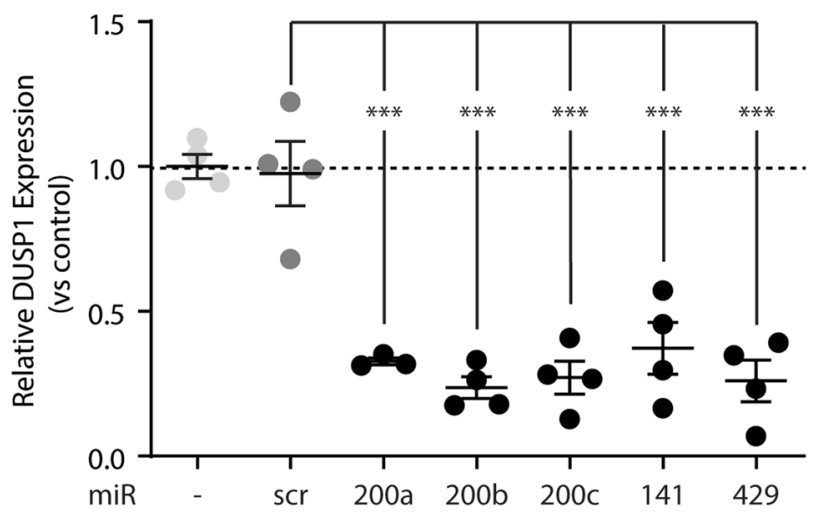

d
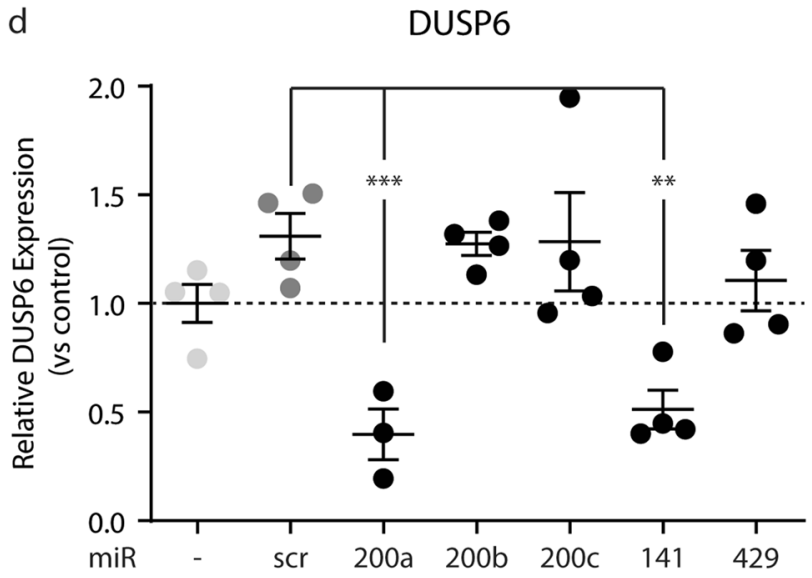

Figure 5. miRNA-200 family members regulated DUSP-1 and DUSP-6 expression. Luciferase reporter assays for microRNA binding were performed for the 3'UTR of DUSP-1 in COS7 cells with ectopic expression of miR-200a, -200b, -200c, -141, -429 or SCR (a). DUSP-1 expression levels were determined by qPCR in HUVEC with ectopic expression of miR-200b, miR-200a, miR-429, miR-200c, miR-141 or scr, normalized to control. All miRNA-200 family members decrease DUSP-1 expression in HUVEC (b). Luciferase reporter assay identified miR-200a and miR-141 to target DUSP-6 (c) and ectopic expression of miR-200a or miR-141 decreases DUSP-6 expression in HUVEC (d). Data is expressed as mean \pm S.D. of all individual observations. Gene expression data and Luciferase activity measurements were obtained from 4 independent experiments. All data was analyzed by ANOVA followed by Bonferroni post hoc tests. ${ }^{\star} \mathrm{P}<0.05$, ${ }^{* *} \mathrm{P}<0.01,{ }^{* *} \mathrm{P}<0.001$.

only a single microRNA were susceptible to TGF $\beta 1$-induced EndMT (data not shown), however, when miR101, miR-200a and miR-141 were transfected in combination, endothelial cells increased their MAPK7 activity (Fig. 7a; Suppl. Fig. 4) and showed reduced expression levels of EZH2 (Fig. 7b; Suppl. Fig. 4). Corroborating the protective effects of MAPK7 signaling in the preclusion of EndMT ${ }^{5}$, TGF $\beta 1$ stimulation did not decrease VECadherin expression (Fig. 7c,d) nor induce the expression of the mesenchymal marker protein SM22 $\alpha$ (Fig. 7c,e) in endothelial cells transfected with miRs-101/200a and -141. Moreover, the ectopic microRNA expression reduced the TGF $\beta 1$-induced increase in endothelial permeability by $\sim 40 \%$ (Fig. 7f) and precluded the TGF $\beta 1$ induced collagen contraction (Fig. 7g) - two functional adaptations associated with EndMT - and maintained the endothelial angiogenic sprouting capacity (Fig. $7 \mathrm{~h}$ ).

\section{Discussion}

In this study, we show that reciprocity exists between the atheroprotective MAPK7 activation and the expression of histone methyltransferase EZH2 in endothelial cells. The reciprocity is regulated by the MAPK7-induced silencing of EZH2 expression by miR-101 and the EZH2-mediated silencing of the miR-200 family, which increases DUSP-1 and DUSP-6 expression and inhibits MAPK7 activation (Fig. 8). The reciprocity between MAPK7-EZH2 might reflect an autoregulatory feedback loop in endothelial cells that ensures endothelial homeostasis. As such, disturbances in this reciprocity leading to increased EZH2 expression can induce endothelial dysfunction and EndMT. In contrary artery disease- a condition associated with EndMT ${ }^{1,26}$ - the reciprocity between MAPK7 and EZH2 is disturbed, resulting in elevated expression of DUSP-1 and EZH2 and the decreased expression of MAPK7. Restoring the reciprocity by ectopic expression of miR-101/200a/429 precludes EndMT and might offer therapeutic benefit in coronary artery disease. 
a

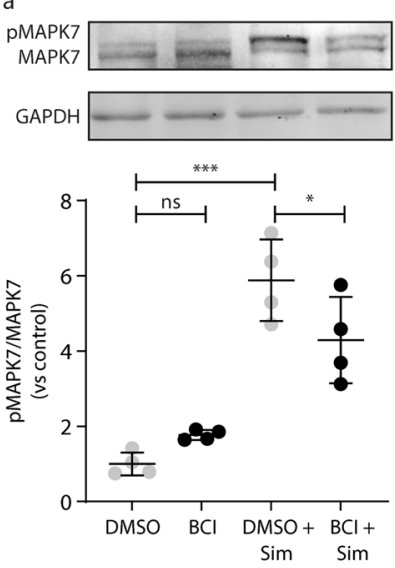

b
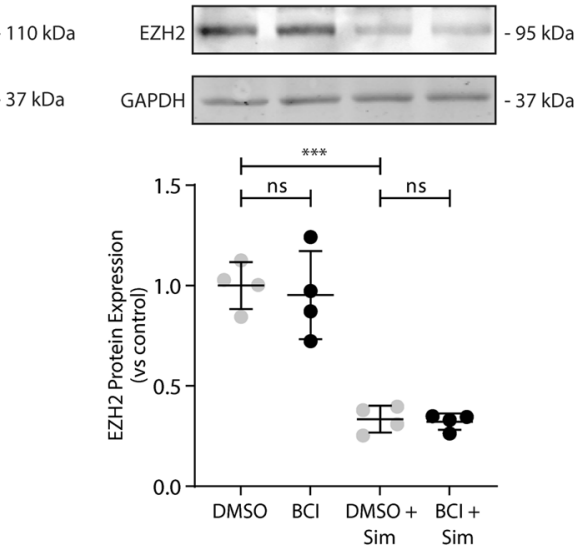
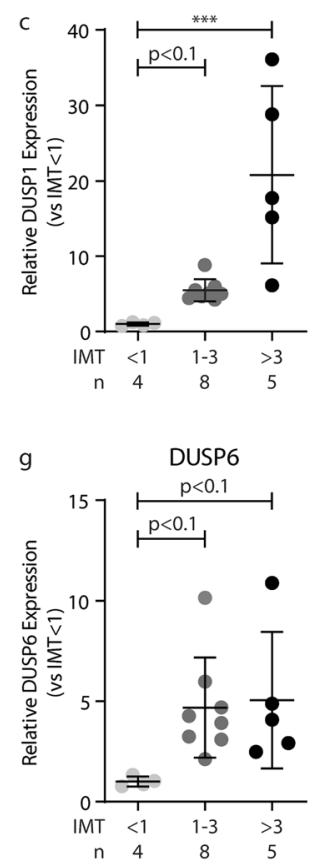

d

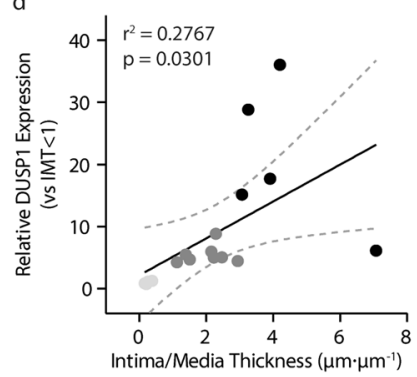

h

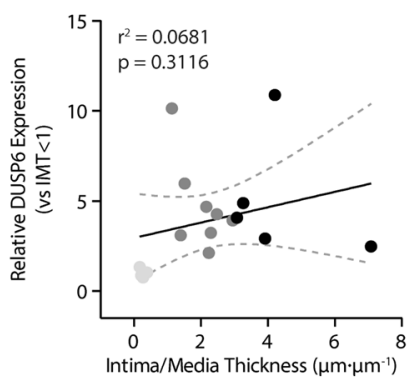

e

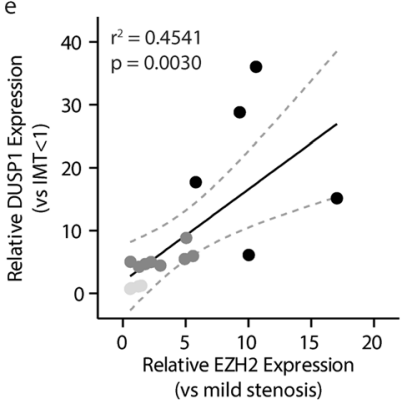

i

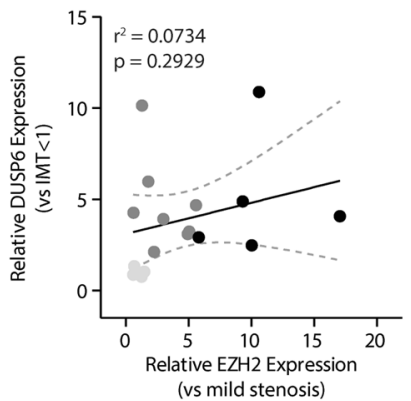

$f$

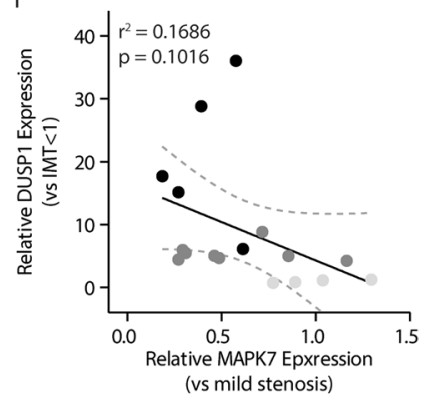

j

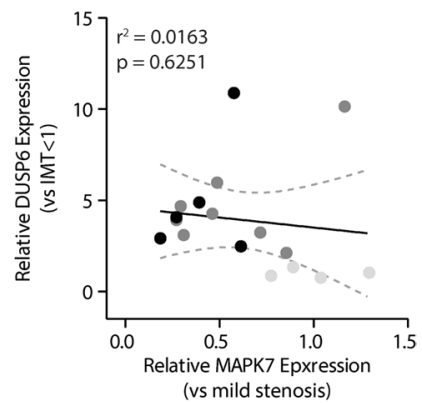

Figure 6. Inhibition of DUSP activity by BCI does not affect MAPK7 activity or EZH2 expression. MAPK7 (a) and EZH2 (b) protein expressions were determined using western blotting, in HUVEC treated with $5 \mu \mathrm{M}$ of the DUSP-1/6 small molecule inhibitor BCI or co-treated with BCI and $10 \mu \mathrm{M}$ Simvastatin for $24 \mathrm{~h}$. Data was normalized to vehicle control cells. DUSP-1 expression levels were determined in human coronary artery disease samples by qPCR and normalized to IMT $<1$ (c). DUSP-1 expression increases with increasing IMT (d) and is associated to EZH2 expression levels (e). DUSP-1 levels tend to show a negative correlation with MAPK7 levels in coronary artery disease (f). DUSP-6 expression levels were determined by qPCR and normalized to IMT $<1$ (g). DUSP-6 expression is elevated in coronary artery disease but does not associate to the severity of disease (h), the level of $E Z H 2$ expression (i), nor the level of $M A P K 7$ expression (j). Sim = Simvastatin $(10 \mu M)$. Data is expressed as mean \pm S.D. of all individual observations. Cell culture data was obtained from 4 independent experiments, whereas human in vivo data was derived from $n=4-8$ samples per group. Data from multiple groups were analyzed by ANOVA followed by Bonferroni post hoc tests. Correlations were performed using Pearson correlation. ${ }^{* *} \mathrm{p}<0.01,{ }^{* *} \mathrm{p}<0.001$.

EndMT contributes to intimal hyperplasia during coronary artery disease ${ }^{1-5}$, wherein MAPK7 signaling plays a protective role ${ }^{5,16}$. EndMT can be induced by hypoxia, inflammatory and fibrogenic signaling ${ }^{6,27}$. Transforming growth factor beta (TGF $\beta$ ) induces EndMT canonically through the activation of downstream mediators Smad2/3, which culminates in the activation of the EndMT transcription factors Snail, Slug and Twist $1{ }^{6}$. MAPK7 inhibits EndMT ${ }^{5,28}$, potentially via the increased expression of inhibitory Smad $7^{29,30}$ and ID proteins ${ }^{31}$ or the repression of TGF control elements in the promoter region of mesenchymal genes ${ }^{32}$. Yet, during intimal hyperplasia the signaling activity of MAPK7 is rapidly lost ${ }^{28}$.

DUSP-1 and DUSP-6 expression levels are elevated in a number of cardiovascular diseases and DUSP-1 deficient mice are protected from atherosclerosis development ${ }^{33,34}$. The elevated expression of DUSPs might explain the loss in protective MAPK7 signaling activity during coronary artery disease. Yet we could not substantiate this 
a

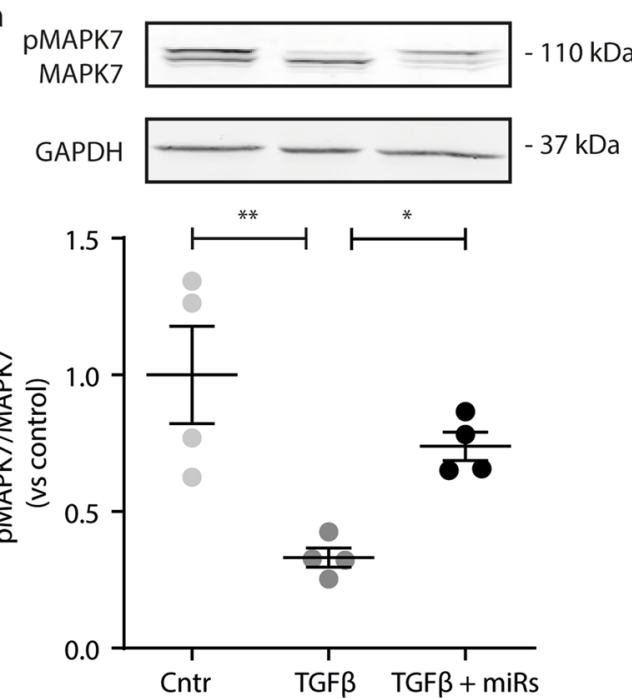

b

$\mathrm{EZH} 2$

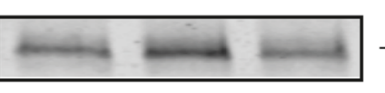

$95 \mathrm{kDa}$

GAPDH
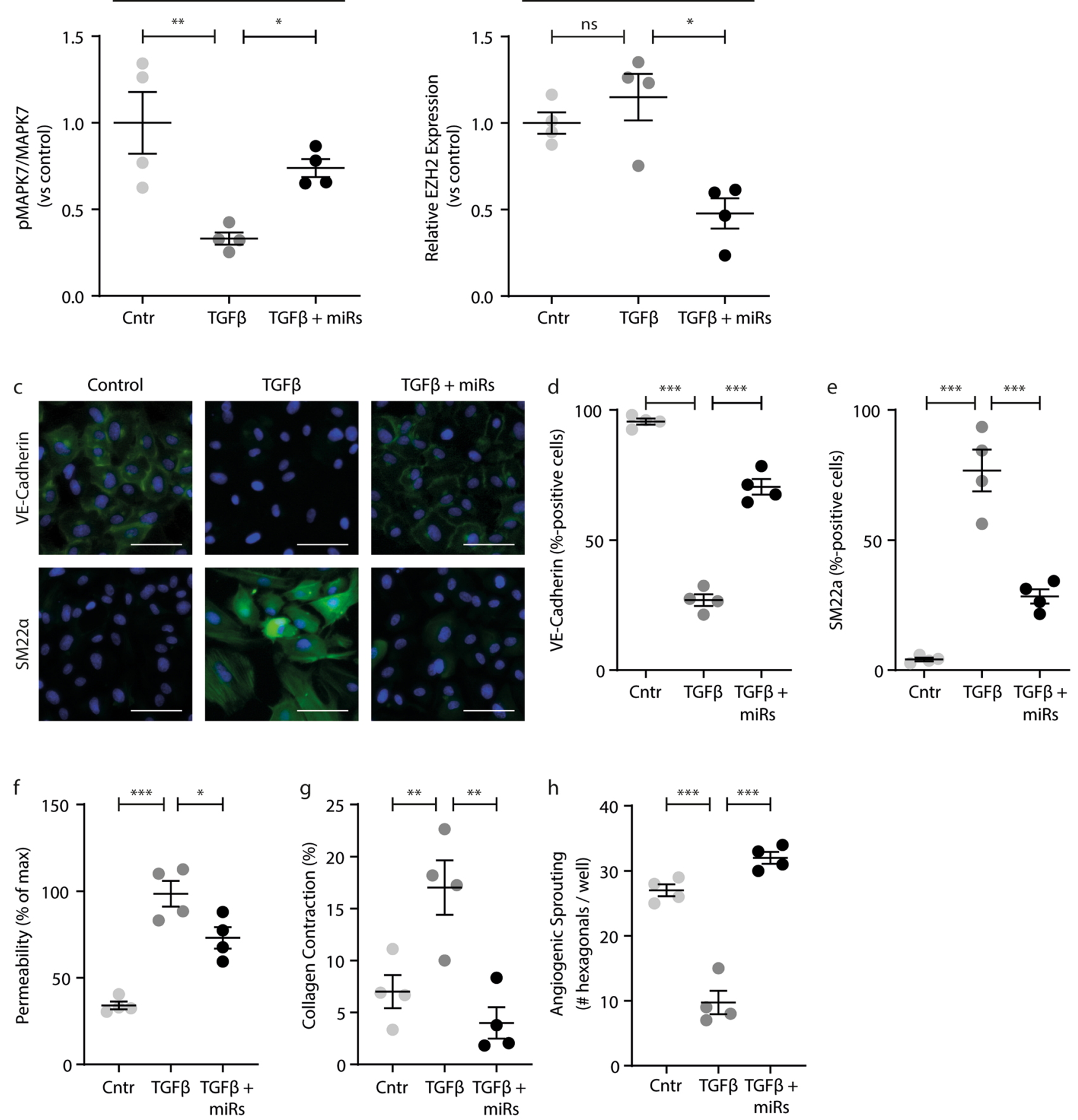

Figure 7. Ectopic expression of miRNA-101, miRNA-141 and miRNA-200a inhibits endothelial dysfunction and EndMT. MAPK7 (a) and EZH2 (b) protein expressions were determined using western blotting, in HUVEC treated with $10 \mathrm{ng} / \mathrm{ml} \mathrm{TGFb1}$ with ectopic expression of miRs-101, -200a and -141 and normalized to untreated control cells. The expression of VE-Cadherin $(\mathbf{c}, \mathbf{d})$ and SM22a $(\mathbf{c}, \mathbf{e})$ were assessed by immunofluorescence and quantified using TissueFaxs analyses. Endothelial cell permeability was assessed using transwell FITCdextran leakage (f) and collagen gel contraction (g) was assessed as a mesenchymal cell function. The angiogenic sprouting behavior of endothelial cells was assessed using the Matrigel assay (h). Data is expressed as mean \pm S.D. of all individual observations. Cell culture data was obtained from 4 independent experiments. Data from multiple groups were analyzed by ANOVA followed by Bonferroni post hoc tests. ${ }^{*} \mathrm{p}<0.05,{ }^{* *} \mathrm{p}<0.01$, ${ }^{* * *} \mathrm{p}<0.001$. Scale bar $=20 \mu \mathrm{m}$. 


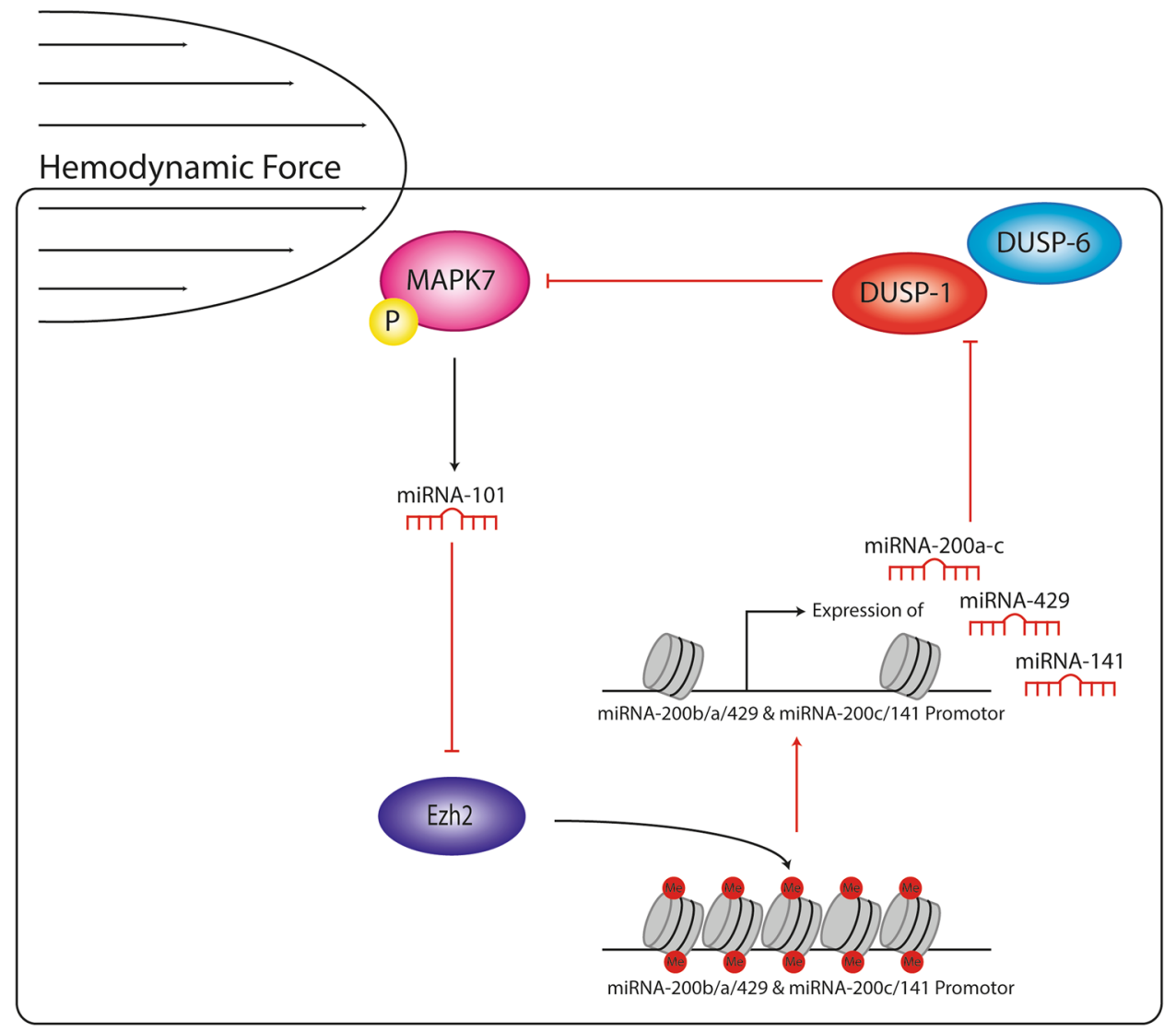

Figure 8. Reciprocal signaling between MAPK7 activity and EZH2 expression in endothelial cells. This reciprocity is regulated by the MAPK7-induced silencing of EZH2 expression by miR-101 and the EZH2mediated silencing of the miR-200 family, which increases DUSP-1 and DUSP-6 expression and inhibits MAPK7 activation. The reciprocity between MAPK7-EZH2 might reflect an autoregulatory feedback loop in endothelial cells that ensures endothelial homeostasis.

hypothesis as pharmacological inhibition of DUSP-1/6 in our experiments did not activate MAPK7 signaling, which may also depend on the availability of ATP and scaffolding proteins, and the activity of upstream MAPK kinases $^{35}$. Besides, the regulation of MAPK7 activity by DUSPs is more complex than direct inactivation. Indeed, DUSP activity is greatly affected by posttranslational modifications ${ }^{36-40}$. For instance, DUSP activity is enhanced by its acetylation ${ }^{37,38}$ or phosphorylation ${ }^{36}$, whereas its oxidation reduces its activity ${ }^{39,40}$. In our experiments, we did not investigate the posttranslational modifications of DUSP1, and in the absence of a response to the small molecule BCI, it is tempting to speculate that the prerequisites for DUSP1 activity were not met in our cell culture model. Moreover, the family of DUSPs contains over twenty members with overlapping substrate specificity ${ }^{41}$, that are not all inhibited by the addition of $\mathrm{BCI}^{42}$. It is therefore conceivable that alternative DUSPs maintain the inhibition of MAPK7 activity in the presence of BCI as a compensatory mechanism. Nonetheless, the expression of DUSP-1 is associated with an increasing IMT, and a decreased expression of MAPK7 in coronary artery disease.

The expression of DUSP- 1 and -6 is associated with high expression of EZH2 in various oncology's ${ }^{23,24}$, albeit by a currently unknown mechanism. We found that EZH2 silences the expression of the microRNA-200 family, which posttranscriptionally regulate the expression of DUSP-1 and DUSP-6. The loss of EZH2 expression by fluid shear stress therefore might increase the expression of miR-200 family members and decrease the expression of the DUSPs culminating in atheroprotective MAPK7 activation. Interestingly, the endothelial cell-specific overexpression of miR-200b precludes EndMT and alleviates diabetic cardiomyopathy in mice ${ }^{43}$. In coronary artery disease, EZH2 expression levels are elevated and high EZH2 expression is associated with endothelial dysfunction ${ }^{18,19}$. In combination, our current data might explain these observations and unifies them into a single mechanism, linking endothelial mechanotransduction to the epigenetic regulation of MAPK7 activity, potentially through DUSP-1 and DUSP-6. This double negative feedback loop might resemble a sensitive autoregulatory mechanism that ensures endothelial homeostasis, which when disturbed culminates in EndMT and possibly coronary artery disease.

It should be noted here, that the downstream effects of EZH2 and MAPK7 on atherogenesis may be much broader than the reciprocal regulation of their expression and activation. Indeed, in earlier work we used RNA sequencing and found that high EZH2 expression coincides with endothelial cell proliferation and 
RNAi-mediated silencing of EZH2 results in endothelial quiescence ${ }^{18}$. Using a similar approach, others reported that high EHZ2 associates with a reduced angiogenic potential and inflammatory activation of endothelial cells ${ }^{19}$. Similarly, the endothelial-specific genetic deletion of MAPK7 aggravates atherogenesis by the induction of endothelial cell inflammatory activation and loss of atheroprotective NO synthesis ${ }^{16}$ and the induction of EndMT ${ }^{5}$. Thus, in future perspective it would be of high interest to validate and extend our current findings in atherosclerosis-prone mouse models (e.g. APOE-deficient or LDLR-deficient mice on a cholesterol-rich diet) wherein MAPK7 and EZH2 expression can be perturbed specifically in the endothelium. Such studies would benefit from the use of transcriptomic, proteomic or "multi-omics" approaches to unravel the multitude of targets and the principle downstream pathways EZH2 and MAPK7 regulate in the atherosclerotic endothelium.

Study limitations. We acknowledge that our study is not without limitations. First, we only included unique 17 coronary artery samples from 10 subjects, which may be a limited number if confounders as hypertension, diabetes or smoking status are to be analyzed. In our study, we chose to stratify these coronary artery samples based on IMT (as a surrogate marker of disease severity) to investigate if the expression of MAPK and EZH2 are "disease-state" specific, and thereby ignored interindividual variation, which may be caused by common risk factors $^{44,45}$ or genetic susceptibility ${ }^{46,47}$. In our study, mean age, hypertension, diabetes and smoking status did not differ between stratified groups and no subject was overrepresented in any group. Using this approach, we show that there is a disbalance in MAPK7 activity and EZH2 expression in coronary artery disease and that this disbalance is perturbed with increasing IMT. In future perspective, it would be highly interesting to investigate how common risk factors affect the disbalance between MAPK7 activity and EZH2 expression in larger cohort studies. In addition, and since polymorphisms in MAPK $7^{48-50}$ and EZH $2^{51-54}$ are identified, it would be highly interesting to investigate whether these polymorphisms associate to a higher cardiovascular risk.

Second, transcriptional expression was performed on of whole artery sections, meaning that the observed differences in MAPK7 and EZH2 expression are not necessarily derived from endothelial cells, but may reflect a significant change in other cell numbers, e.g. inflammatory and smooth muscle cells. Indeed, the cellular composition of a coronary artery lesion is dynamic and may change with disease progression ${ }^{55,56}$. Although MAPK7 expression appears endothelial cell-restricted in porcine and mouse arteries ${ }^{28}$, EZH2 is expressed by a plethora of cells. In vivo validation of our current findings by localization techniques such as double immunofluorescence would have to be performed in order to show endothelial specificity of the reported results. Moreover, the endothelial cell-specific deletion of MAPK7 and EZH2 in atherosclerosis-prone mice would further emphasize their relevance to atherogenesis in future perspective.

Third, for the mechanistic experiments detailed here, we used HUVEC rather than primary human coronary artery endothelial cells (HCAEC), and their distinct origin might interfere on our observations. Although the endothelium harbors a larger heterogeneity in vivo between endothelial cells from distinct vascular beds ${ }^{57,58}$, cultured endothelial cells rapidly lose the expression of vascular bed-specific markers ${ }^{57-60}$. HUVEC maintain expression of generalized endothelial function in cell culture, including shear stress responsiveness ${ }^{5,61}$. Hence, HUVEC are commonly used for mechanistic studies of endothelial cell behavior ${ }^{62}$. Moreover, HUVEC and HCAEC show similar in vitro responses to LSS stimulation ${ }^{61}$.

\section{Conclusion}

To summarize, in endothelial cells there is reciprocity between MAPK7 activity and the expression of EZH2. This reciprocity is regulated in part by a complex mechanism involving microRNAs and the regulation of phosphatase activity (Fig. 8). Dysregulation in the reciprocity between MAPK7 activation and EZH2 expression is associated to the induction of EndMT and the severity of coronary artery disease. These insights contribute to a better understanding of the molecular and epigenetic mechanisms that underlie endothelial homeostasis, the induction of EndMT during coronary artery disease and might represent a new target for therapy.

\section{Materials and methods}

Human coronary artery samples. Seventeen unique human coronary artery samples were obtained from autopsy specimens from 10 patients (age 59.1 \pm 2.6 years, range 39-69) that died from an acute coronary episode at the Heart Institute (InCor), Sao Paulo, Brazil. Hypertension was present in 9 subjects, and diabetes in 6. Five individuals were active smokers. Coronary artery samples were stratified based on their respective intimamedia thickness (IMT) prior to further analyses. Stratified sample groups never contained more than one sample per patient. Next-of-kin gave informed consent and the investigation was performed according to institutional guidelines and approved by the Institutional CAPPesq Ethics Committee (InCor, Sao Paulo \#SDC 3723/11/141 and \#CAPPesq 482/11) and the Declaration of Helsinki ${ }^{28}$. All experimental protocols were approved by the Institutional CAPPesq Ethics Committee (InCor, Sao Paulo, Brazil). During necropsy each dissected coronary artery was fixed in neutral-buffered formalin with constant perfusion at a quasi-normal perfusion pressure before paraffin embedding.

Determination of intima-media thickness. Four micron-thick sections were prepared from human coronary artery samples and deparaffinized using Xylol and rehydrated using a series of EtOH solutions of decreasing concentration. Samples were stained in Verhoeff's solution (92 mM hematoxylin, $137 \mathrm{mM} \mathrm{FeCl}$, $27 \mathrm{mM} \mathrm{KI}, 4 \mathrm{mM} \mathrm{I}_{2}$ in 55\% EtOH) at room temperature for $1 \mathrm{~h}$. Samples were differentiated in $\mathrm{FeCl}_{3}(123 \mathrm{mM}$

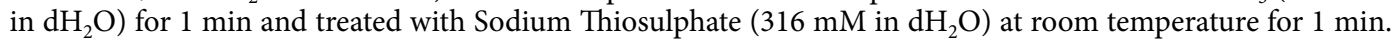
Samples were dehydrated using increasing concentrations of EtOH and cleared in $100 \%$ xylene. Samples were mounted in Permount resinous mounting medium. The intimal thickness was determined as the distance between the inner elastic lamina and the lumen, and the medial thickness was determined by measuring the 
distance between the inner elastic lamina and the outer elastic lamina at ten sites within one sample. Intimal/ Medial thickness was calculated by dividing the average intimal thickness by the average medial thickness ${ }^{28}$.

Endothelial cell culture and uniform laminar shear stress experiments. Human umbilical vein endothelial cells (HUVEC, Lonza \#C2519) were cultured in endothelial cell culture medium (ECM) as described previously ${ }^{5,63}$. EndMT was induced by the addition of $10 \mathrm{ng} / \mathrm{ml}$ TGF $\beta 1$ to the culture medium as described before $^{5,64}$. For shear stress experiments, HUVEC $\left(60000 \mathrm{cells} / \mathrm{cm}^{2}\right)$ were seeded on $0.1 \%$ gelatin-coated $\mu$-Slides I 0.4 Luer (Ibidi GmbH, Martinsried, Germany) and allowed to adhere under standard culture conditions overnight. Slides with a confluent endothelial cell monolayer were exposed to uniform laminar shear stress (20 dyne/ $\mathrm{cm}^{2}$ ) for $24 \mathrm{~h}$. Where indicated, $10 \mu \mathrm{M}$ of the small molecule inhibitor of MAPK7 (BIX02189, SelleckChem, Munich, Germany) $)^{14}, 5 \mu \mathrm{M}$ of the small molecule inhibitor of DUSP-1/6 (BCI, Axon Medchem, Groningen, The Netherlands) or $10 \mu \mathrm{M}$ simvastatin (SelleckChem, Munich, Germany) ${ }^{65}$ was applied.

Viral transduction of endothelial cells. pLKO.1-shEZH2 and pLKO.1-SCR were kindly provided by Prof.dr. J.J. Schuringa (dept. Hematology, UMCG). HEK293 cells were co-transfected with pLKO.1-shEZH2 or pLKO.1-SCR, pVSVG (envelope plasmid) and pCMV-R8.91 (gag-pol 2nd generation packaging plasmid) using Endofectin-Lenti (Gene Copoeia, Rockville, MD, USA). At 48- and 72-h post-transfection, viral supernatants were collected.

A retroviral construct encoding the constitutively active rat MEK5- $\alpha 1$ (pBabePuro-MEK5D) and empty vector controls were kindly provided by Prof.dr. M. Schmidt (Dept. Dermatology, University Würzburg, Germany). Retroviral transduction of HUVEC was performed as detailed before ${ }^{66}$. In brief, virus-producing Phoenix cells were cultured until 70\% confluency, after which basal medium was replaced by ECM after which viral supernatants were collected twice at $24 \mathrm{~h}$ intervals.

Viral supernatants were supplemented with polybrene ( $6 \mu \mathrm{g} / \mathrm{ml}$; Sigma, St.Louis, MO) and applied to $30 \%$ confluent HUVEC for two consecutive rounds of $24 \mathrm{~h}$ exposure. Transduced HUVECs were passaged twice, and transduced cells were selected by puromycin ( $4 \mu \mathrm{g} / \mathrm{ml}$; Invitrogen, Carlsbad, CA, USA).

MicroRNA transfections in endothelial cells. HUVEC or COS7 cells were seeded in antibiotic free medium at a density of $20,000 / \mathrm{cm}^{2}$. Cells were transfected with $50 \mathrm{pmol}$ of microRNA mimics (miR-101 (\#PM11414), miR-200a (\#PM10991), miR-200b (\#PM10492), miR-200c (\#PM11714), miR-141 (\#PM10860), miR-429 (\#PM10221) or scrambled control (\#AM17110, all Ambion/Life Technologies, Carlsbad, CA) using the siRNA reagent system (Santa Cruz, \#sc-45064, Santa Cruz, CA) according to manufacturer's instructions.

Immunofluorescence. Immunofluorescence analysis was performed for the endothelial cell marker VECadherin (R\&D \#9381, Minneapolis, MN) and the mesenchymal cell marker SM22a (Abcam \#14106, Cambridge, UK) as previously described ${ }^{64}$.

Immunoblotting. Cells were harvested in RIPA buffer (Thermo Fisher Scientific, Waltham, MA) supplemented with $1 \% \mathrm{v} / \mathrm{v}$ protease inhibitor cocktail (Sigma Aldrich, St Louis, MO) and 1\% v/v phosphatase inhibitor cocktail (Sigma Aldrich, St Louis, MO). Samples were sonicated and protein concentration was determined with a DC protein assay (Bio-Rad, Hercules, CA). Equal amounts of protein were separated by electrophoresis on $10 \%$ polyacrylamide gels after which proteins were blotted onto nitrocellulose membranes using the semi dry Transblot Turbo system (Bio-Rad, Hercules, CA). Membranes were trimmed to conserve antibodies and blocking reagent, and were blocked with Odyssey Blocking buffer (Li-COR Biosciences, Lincoln, NE) at RT for $1 \mathrm{~h}$, and incubated with antibodies against $\beta$-actin (1:2000, Cell Signaling, Danvers, MA, USA), EZH2 (1:1000, Cell Signaling, Danvers, MA, USA), MAPK7 (1:1000, Merck Millipore, Billerica, MA, USA), MKP-1(DUSP-1, 1:1000, Abcam \#195261) or MKP-3 (DUSP-6, 1:500, Santa Cruz, \#sc377070) at 4 C overnight. Membranes were washed in TBS Tween $(0.1 \%)$ and developed using IrDye-conjugated antibodies to rabbit IgG $(1: 10,000$, \#92668021), mouse IgG (1:10,000, \# 926-32210, both Li-COR Biosciences) or AP-conjugated antibodies to rabbit IgG (1:2000, \#7054S, Cell Signaling) at RT for $1 \mathrm{~h}$. Protein detection was done using the Odyssey Infrared Imaging System (Li-COR Biosciences). The development of AP-conjugated antibodies, membranes were incubated with AP-detection buffer ( $100 \mathrm{nM} \mathrm{NaCl}, 100 \mathrm{mM}$ Tris, $50 \mathrm{mM} \mathrm{MgCl}_{2}$, $\mathrm{pH}$ 9.5) supplemented with nitro-blue tetrazolium chloride (NBT) $(330 \mu \mathrm{g} / \mathrm{mL})$ and 5-bromo-4-chloro-3'-indolyphosphate p-toluidine salt (BCIP) (165 $\mu \mathrm{g} /$ $\mathrm{mL}$ ). Densitometry analysis was performed using Totallab 120 (Nonlinear Dynamics, Newcastle upon Tyne, England). Unprocessed images of individual immunoblots used for quantification are available in the online data supplement.

RNA isolation and transcript analysis. Sections of whole arterial thickness were deparaffinated using xylol and rehydrated prior to homogenization in TRIzol (Invitrogen Corp, CA, USA). Cell cultures were lysed directly in TRIzol. RNA was isolated using the TRIzol reagent according to the manufacturer's protocol. RNA concentration and purity were assessed using UV spectrometry (Nanodrop 1000, Thermo Scientific MA, USA) and RNA integrity validated on $1 \%$ agarose gels. For gene expression analysis, cDNA synthesis was performed using RevertAid ${ }^{\text {TM }}$ First Strand cDNA Synthesis Kit (Thermo Scientific, MA, USA), according to the manufacturer's protocol. For microRNA transcript analysis, $10 \mathrm{ng}$ of total RNA was reversely transcribed using the ABI Taqman microRNA reverse transcription kit (\#4366597, ThermoFisher Scientific) according to manufactures instructions using 1.0 $\mu \mathrm{M}$ microRNA-specific stemloop primers (Table 1). For all transcript analyses, the cDNA was amplified on a VIIA7 thermal cycling system (Applied Biosystems, Carlsbad, CA) in a reaction contain- 


\begin{tabular}{|l|l|}
\hline Gene & Sequence \\
\hline$m i R-101$ & $\begin{array}{l}\text { Stem loop: GTCGTATCCAGTGCAGGGTCCGAGGTATTCGCACTGGATACGACTTCAGTTA } \\
\text { Sense: TGCGGTACAGTACTGTGAT }\end{array}$ \\
\hline$m i R-141$ & $\begin{array}{l}\text { Stem loop: GTCGTATCCAGTGCAGGGTCCGAGGTATTCGCACTGGATACGACCCATCTTTAC } \\
\text { Sense: TGCGGTAACACTGTCTG }\end{array}$ \\
\hline$m i R-200 a$ & $\begin{array}{l}\text { Stem loop: GTCGTATCCAGTGCAGGGTCCGAGGTATTCGCACTGGATACGACACATCGTT } \\
\text { Sense: TGCGGTAACACTGTCTGGT }\end{array}$ \\
\hline$m i R-200 b$ & $\begin{array}{l}\text { Stem loop: GTCGTATCCAGTGCAGGGTCCGAGGTATTCGCACTGGATACGACTCATCATTAC } \\
\text { Sense: TGCGGTAATACTGCCTG }\end{array}$ \\
\hline$m i R-200 c$ & $\begin{array}{l}\text { Stem loop: GTCGTATCCAGTGCAGGGTCCGAGGTATTCGCACTGGATACGACCCAAACACTG } \\
\text { Sense: TGCGGCGTCTTACCCAG }\end{array}$ \\
\hline$m i R-429$ & $\begin{array}{l}\text { Stem loop: GTCGTATCCAGTGCAGGGTCCGAGGTATTCGCACTGGATACGACACGGTTTTAC } \\
\text { Sense: TGCGGTAATACTGTCTG }\end{array}$ \\
\hline$U 6$ & $\begin{array}{l}\text { Stem loop: GTCGTATCCAGTGCAGGGTCCGAGGTATTCGCACTGGATACGACAAAAATATGG } \\
\text { Sense: TGCGGCTGCGCAAGGATGA }\end{array}$ \\
\hline$U 24$ & $\begin{array}{l}\text { Stem loop: GTCGTATCCAGTGCAGGGTCCGAGGTATTCGCACTGGATACGACTGCATCAGCG } \\
\text { Sense: TGCGGTGCAGATGATGTAA }\end{array}$ \\
\hline & Antisense: GTGCAGGGTCCGAGGT \\
\hline
\end{tabular}

Table 1. Primer sequences for microRNA expression analysis.

\begin{tabular}{|l|l|}
\hline Gene & Sequence \\
\hline DUSP-1 & $\begin{array}{l}\text { Sense: TGGGTACATCAAGTCCATCTGA } \\
\text { Antisense: GCAAAAAGAAACCGGATCAC }\end{array}$ \\
\hline DUSP-6 & $\begin{array}{l}\text { Sense: GACGCTCGCTGTTTGTATCC } \\
\text { Antisense: GACTCAGCCTCGCACACC }\end{array}$ \\
\hline$E Z H 2$ & $\begin{array}{l}\text { Sense: GCGAAGGATACAGCCTGTGCACA } \\
\text { Antisense: AATCCAAGTCACTGGTCACCGAAC }\end{array}$ \\
\hline GAPDH & $\begin{array}{l}\text { Sense: AGCCACATCGCTCAGACAC } \\
\text { Antisense: GCCCAATACGACCAAATCC }\end{array}$ \\
\hline MAPK7 & $\begin{array}{l}\text { Sense: CCTGATGTCAACCTTGTGACC } \\
\text { Antisense: CCTTTGGTGTGCCTGAGAAC }\end{array}$ \\
\hline
\end{tabular}

Table 2. Primer sequences for gene expression analysis.

ing $0.6 \mu \mathrm{M}$ primers (Table 2) using SYBR Green chemistry (Bio-Rad, VA, USA). Cycle threshold $\left(\mathrm{C}_{\mathrm{T}}\right)$ values for individual reactions were determined and normalized against GAPDH/ACTB (gene transcript analysis) or RNU6 (microRNA transcript analysis). All cDNA samples were amplified in triplicate. Relative expression was calculated using the $\Delta \mathrm{C}_{\mathrm{T}}$ method. Data are presented as fold change compared with control.

3'UTR binding assays. 3'UTR fragments were isolated, purified and cloned in the psiCKECK-2 reporter vector as described previously ${ }^{64}$. Specific primers for the EZH2-3'UTR (sense 5'-CATCTGCTACCTCCTCCC CC-3', antisense 5'-GACAAGTTCAAGTATTCTTT-3'), DUSP-1-3'UTR (sense 5'-AAGGCCACGGGAGGT GAGGC-3', antisense 5'-CAATAGAAATGCCATAATTT-3'), and DUSP-6-3'UTR (sense 5'-AAGACCCCA CACCCCTCCTT-3', antisense 5'-CAATAGCCAAAATAGTTATT-3', all 0.6 $\mu \mathrm{M}$, Biologio, Leiden, The Netherlands) were used to isolate the 3'-UTR fragments from a cDNA pool of various human tissues.

COS7 cells were transfected with $100 \mathrm{ng}$ UTR reporter plasmid and $50 \mathrm{pmol}$ microRNA mimics as detailed above. $48 \mathrm{~h}$ post-transfection, luciferase activity was assayed using the DualGlo Luciferase assay system (Promega, Madison, WI) and recorded for $500 \mathrm{~ms}$ on a Luminoskan ASCENT (Thermo Scientific, Waltham, MA) according to manufacturer's instructions. Relative luciferase activity was calculated by dividing the luminescence from Renilla luciferase activity by the luminescence from firefly luciferase activity and normalized to control samples.

chromatin-immunoprecipitation (ChIP) and assessment of histone modifications. Cells were harvested using accutase, pelleted and the chromatin crosslinked using 1\% formaldehyde (37\% F1268 SigmaAldrich) for $8 \mathrm{~min}$. Crosslinking activity was quenched using $125 \mathrm{mM}$ glycine (104201 Merck). Cell pellets were lysed on ice with SDS lysis buffer (1\% SDS, $50 \mathrm{mM}$ Tris $\mathrm{HCl} \mathrm{pH} \mathrm{8.0,10} \mathrm{mM} \mathrm{EDTA)} \mathrm{supplemented} \mathrm{with} \mathrm{freshly}$ added $100 \mathrm{mM}$ protease inhibitor cocktail (Sigma Aldrich P8340) for $15 \mathrm{~min}$. The chromatin was fragmented by Biorupter (Diagenode, Seraing, Belgium) with five cycles of (30' ON/OFF). The sonicated sample was centrifuged and chromatin containing supernatant was kept for further analysis. The chromatin was diluted 10 times with RIPA buffer $(0.1 \%$ SDS, $0.1 \%$ Sodium deoxycholate, $1 \%$ Triton-X100, $1 \mathrm{mM}$ EDTA, $10 \mathrm{mM}$ Tris-HCl pH 7.5, $140 \mathrm{mM} \mathrm{NaCl}, 0.5 \mathrm{mM}$ EGTA) supplemented with $100 \mathrm{mM}$ protease inhibitor cocktail. Immunoprecipitation was performed by $4 \mu \mathrm{g}$ H3K27Me3 antibody (Merk Millipore 07-449) or IgG control (Abcam ab46540) added to the 40uL Dynabeads Protein-A (Life technologies, 10002D) coated tubes. Subsequently, the chromatin of $0.8 \times 10^{6}$ cells was added to antibody bound beads and incubated overnight at $4 \mathrm{C}$ while rotating. The beads 


\begin{tabular}{|c|c|c|c|}
\hline Genomic region & Chr & Sense sequence & Antisense sequence \\
\hline \multicolumn{4}{|l|}{$m i R-200 b / a / 429$} \\
\hline$-2.5 \mathrm{~kb}$ & 1 & GGAGGAGCTGGTGTGTTCTC & CAAAGCCGCCATTTCACC \\
\hline$-2.0 \mathrm{~kb}$ & 1 & GCGGTGATGATTAACCCAAC & GTGGCCACAGGTCAAGAAAT \\
\hline$-1.5 \mathrm{~kb}$ & 1 & GGTGAGAACGCAATGACTGA & CTCCCACTGCCAGGTTCA \\
\hline$-1.0 \mathrm{~kb}$ & 1 & TTGGAGGAGGAGACTGGAAC & AGTTTTCTGGCACCTTCCAC \\
\hline$-0.5 \mathrm{~kb}$ & 1 & GACCAGCAGACACACAAACC & GACСССТСТСССАТGCTG \\
\hline TSS & 1 & TACTGAGCTTCCCAGCGAGT & AATGCTGCCCAGTAAGATGG \\
\hline$+0.5 \mathrm{~kb}$ & 1 & GAGCAGGACCCAACAGAGG & CAGGAGGGAAGATGGCTGT \\
\hline \multicolumn{4}{|l|}{ miR-200c/141 } \\
\hline$-2.5 \mathrm{~kb}$ & 12 & CACCTCCAGGTTCCAGCTAC & AAATGCTTCCACAGGGTCAG \\
\hline$-2.0 \mathrm{~kb}$ & 12 & ACAGGGTGGTTGTGAAAAGC & CGCCACGGTAAAATGAGAAT \\
\hline$-1.5 \mathrm{~kb}$ & 12 & CATGTCAGGAGTGGGGTTTC & CTTTGGGACCTGTGCTGTCT \\
\hline$-1.0 \mathrm{~kb}$ & 12 & GACTCCACTGAGGGCTGTG & TGAAGTTACCCACCGCTACC \\
\hline$-0.5 \mathrm{~kb}$ & 12 & GCCTAGAGGAGTGGCCAAG & GGTGTGTCCTCCTGCCATAG \\
\hline TSS & 12 & AGGGCTCACCAGGAAGTGT & AGGATCCCTGCGGAAAAG \\
\hline$+0.5 \mathrm{~kb}$ & 12 & CCCTGTAGCAACTGGTGAGC & GGGAGCCATCTTTACCAGAC \\
\hline \multicolumn{4}{|l|}{ miR-101-1 } \\
\hline$-2.5 \mathrm{~kb}$ & 1 & CCACAGGCCTGGTTGTAGAT & AATCATTGGCCTTGGTGAAG \\
\hline$-2.0 \mathrm{~kb}$ & 1 & TGGGTAGAGCAGAGGGAAGA & ATCCTTCATTGTGCCAGTCC \\
\hline$-1.5 \mathrm{~kb}$ & 1 & TGACCGCAGACTGAGACTCTT & GCCAGGGAGAGAAAAACCAT \\
\hline$-1.0 \mathrm{~kb}$ & 1 & TGGAGGTTGAAGATGTGTGC & CTGGTCTTGACCTCCTGAGC \\
\hline$-0.5 \mathrm{~kb}$ & 1 & TGACAGCAGCAGCAATAACA & CCAGCTTACTGAAGTGAAGAAAGA \\
\hline TSS & 1 & TTCTTCCTGGGTACGGTGAG & CCGACACAGTGACTGACAGG \\
\hline$+0.5 \mathrm{~kb}$ & 1 & ACTGACTGTGCCTCCCTGAC & TGAGCACTTTGAAGACAGGA \\
\hline \multicolumn{4}{|l|}{ DUSP-1 (MKP-1) } \\
\hline$-2.5 \mathrm{~kb}$ & 5 & CCTCACCCCTGCTCTTTATG & ATGGCTTGCAGTGACCTTCT \\
\hline$-2.0 \mathrm{~kb}$ & 5 & CAGCAAGGGAGGAGAGAGAA & GCCTGGTGACAGAGCAAGAC \\
\hline$-1.5 \mathrm{~kb}$ & 5 & GCCTCGCTTAGCTTGTGTGT & CACTCCATGCCCTGAACTTT \\
\hline$-1.0 \mathrm{~kb}$ & 5 & GCAGTGGATTCCAGGGTTT & GAAAGGGATGGAGAAGCTCA \\
\hline$-0.5 \mathrm{~kb}$ & 5 & GCTTCCTGTGCTTTTGCATAC & CCCCAGTAGTGTGGTTCTGG \\
\hline TSS & 5 & CGCTTTTGGACTGAGAGAGG & CTCGCTGCGAAGGACATT \\
\hline$+0.5 \mathrm{~kb}$ & 5 & AGGGCGTACCTTTGAGGAAG & GTGGTGTTGCTGGACGAG \\
\hline \multicolumn{4}{|l|}{ DUSP-6 (MKP-3) } \\
\hline$-2.5 \mathrm{~kb}$ & 12 & AGGCCTAGGTTGCCAATTTT & AAAATGGTGCGGAGAGGAG \\
\hline$-2.0 \mathrm{~kb}$ & 12 & ATTGGAAGCCGGATGGAG & GCAGGCTTCGGCACTTTTAT \\
\hline$-1.5 \mathrm{~kb}$ & 12 & AATGATTTCTGGGCAAGGAG & GGTCTTGCGGGAGGACTT \\
\hline$-1.0 \mathrm{~kb}$ & 12 & GACCCAAGTTCGCCTTAACC & ACACAGCCTCGGCTAAAAGA \\
\hline$-0.5 \mathrm{~kb}$ & 12 & AGGCAGCTCCTCAATGGATA & TCATCAACACAACCTGTTCCA \\
\hline TSS & 12 & GTCTTGCTGATCGCCATTTC & AGCTCGACCCCCATGATAG \\
\hline$+0.5 \mathrm{~kb}$ & 12 & GGAAGCGAGTGGATTCTGAG & CGCGTGGATTGAAAATACCT \\
\hline
\end{tabular}

Table 3. Primers used for CHIP-qPCR assays.

were washed 3 times with ice cold PBS and the remaining complexes were eluted with $100 \mathrm{nM} \mathrm{NaHCO}_{3}$ and $1 \%$ SDS in PBS. $5 \mathrm{M} \mathrm{NaCl}$ and RNAse (Roche \#11119915001) were added to the eluted samples and incubated at 62 $\mathrm{C}$ to reversing the crosslink for $4 \mathrm{~h}$. $2 \mu \mathrm{L}$ Proteinase K (Roche \#03115828001) was added and incubated at $62 \mathrm{C}$ for $1 \mathrm{~h}$ to liberate the DNA from the histones. DNA fragments were purified using a QIAquick PCR purification kit (Qiagen) according to manufacturers' instructions. Precipitated DNA was analyzed by qPCR using seven sets of primers for each promoter area. (Table 3; all $0.6 \mu \mathrm{M}$ Biolegio) Enrichment of promoter sequences in the precipitate were calculated relative to the percentage of input.

Angiogenic sprouting assay. $10 \mu \mathrm{L}$ Matrigel (BD Corning, 356230) was added into the bottom compartment of $\mu$ slide Angiogenesis (81501, Ibidi GmbH, Martinsried, Germany) and incubated at $37 \mathrm{C}, 5 \% \mathrm{CO}_{2}$ for $1 \mathrm{~h}$. Cells were diluted to $2 \times 10^{6}$ cells $/ \mathrm{ml} .50 \mu \mathrm{l}$ cell suspension was added on top compartment. After $6 \mathrm{~h}$ incubation at $37 \mathrm{C}, 5 \% \mathrm{CO}_{2}$, light microscopy images were obtained, and complete octamer niches were counted by eye. 
Collagen contraction assay. Cells were dissociated using trypsin-EDTA, pelleted and suspended at a concentration of $22.5 \times 10^{6}$ cells $/ \mathrm{ml} \mathrm{ECM.} 45 \mu \mathrm{L}$ cell suspension was added to a collagen solution $(3.3 \mathrm{mg} / \mathrm{ml} \mathrm{rat}$ tail collagen type I (\#354236, BD, San Jose, CA), $100 \mathrm{mM} \mathrm{Na} \mathrm{HPO}_{4}$ and $5 \mathrm{mg} / \mathrm{ml} \mathrm{NaHCO}_{3}$ ) of neutral $\mathrm{pH}$. The cell/collagen mixture was immediately aliquoted into $50 \mu$ droplets and allowed to polymerize at $37 \mathrm{C}, 5 \% \mathrm{CO}_{2}$ for $30 \mathrm{~min}$. Polymerized gels were released and $1 \mathrm{~mL}$ of ECM was added. At time points $\mathrm{t}=0 \mathrm{~h}$ and $\mathrm{t}=24 \mathrm{~h}$, gels were visualized using a regular flatbed scanner and the gel surface area quantified using with Image (NIH). Gel contraction was calculated as the relative reduction in gel surface area at $24 \mathrm{~h}$.

Permeability assay. Cells $\left(5 \times 10^{4} / \mathrm{cm}^{2}\right)$ were cultured on polycarbonate cell culture inserts strips (pore size $0.4 \mu \mathrm{m}$, porosity, $0.9 \times 10^{8} / \mathrm{cm}^{2}$ Fisher Scientific, \#15639536) coated with $0.1 \%$ gelatin for $72 \mathrm{~h}$ to establish a monolayer. Monolayer permeability was assessed by the addition of $5 \mu \mathrm{g} / \mathrm{mL}$ FITC dextran (Sigma) in upper compartment. Fluorescence was measured in the bottom compartment on fluorescence reader at Ex485/Em519 $30 \mathrm{~min}$ after the addition of dextran. Relative permeability levels were calculated using the fluorescence signal of a naked strip ( $100 \%$ permeability) or the fluorescence signal from the culture medium ( $0 \%$ permeable). Permeability was calculated by following formula: Permeability $=\left(\mathrm{Em}_{519}(\operatorname{sample})-\mathrm{Em}_{519}(\mathrm{ECM})\right) / \mathrm{Em}_{519}($ Empty well $)$ $\times 100$.

Data representation of statistical analyses. Data are expressed as mean \pm s.e.m. from at least three independent experiments. Where the mean of two groups were compared, $\mathrm{p}$-values were calculated using student t-tests. Otherwise, p-values were calculated using the one-way analysis of variance (ANOVA) followed by Bonferroni's post-hoc comparisons tests using GraphPad Prism 9 (GraphPad Software, La Jolla, CA, USA). $\mathrm{P}<0.05$ was considered statistically significant.

\section{Data availability}

All data generated or analyzed during this study are included in this published article. Materials, data, and associated protocols are available from the corresponding author on reasonable request without preconditions.

Received: 2 September 2020; Accepted: 4 August 2021

Published online: 07 September 2021

\section{References}

1. Chen, P. Y. et al. Endothelial-to-mesenchymal transition drives atherosclerosis progression. J. Clin. Invest. 125, 4514-4528. https:// doi.org/10.1172/JCI82719 (2015).

2. Evrard, S. M. et al. Endothelial to mesenchymal transition is common in atherosclerotic lesions and is associated with plaque instability. Nat. Commun. 7, 11853. https://doi.org/10.1038/Ncomms11853 (2016).

3. Mahmoud, M. M. et al. TWIST1 integrates endothelial responses to flow in vascular dysfunction and atherosclerosis. Circ. Res. 119, 450-462. https://doi.org/10.1161/CIRCRESAHA.116.308870 (2016).

4. Mahmoud, M. M. et al. Shear stress induces endothelial-to-mesenchymal transition via the transcription factor snail. Sci. Rep. 7, 3375. https://doi.org/10.1038/s41598-017-03532-z (2017).

5. Moonen, J. R. et al. Endothelial-to-mesenchymal transition contributes to fibro-proliferative vascular disease and is modulated by fluid shear stress. Cardiovasc. Res. 108, 377-386. https://doi.org/10.1093/cvr/cvv175 (2015).

6. Souilhol, C., Harmsen, M. C., Evans, P. C. \& Krenning, G. Endothelial-mesenchymal transition in atherosclerosis. Cardiovasc. Res. 114, 565-577. https://doi.org/10.1093/cvr/cvx253 (2018).

7. Markwald, R. R., Fitzharris, T. P. \& Manasek, F. J. Structural development of endocardial cushions. Am. J. Anat. 148, 85-119. https://doi.org/10.1002/aja.1001480108 (1977).

8. Maddaluno, L. et al. EndMT contributes to the onset and progression of cerebral cavernous malformations. Nature 498, $492-496$. https://doi.org/10.1038/nature12207 (2013).

9. Hashimoto, N. et al. Endothelial-mesenchymal transition in bleomycin-induced pulmonary fibrosis. Am. J. Respir. Cell Mol. Biol. 43, 161-172. https://doi.org/10.1165/rcmb.2009-0031OC (2010).

10. Zeisberg, E. M., Potenta, S. E., Sugimoto, H., Zeisberg, M. \& Kalluri, R. Fibroblasts in kidney fibrosis emerge via endothelial-tomesenchymal transition. J. Am. Soc. Nephrol. 19, 2282-2287. https://doi.org/10.1681/ASN.2008050513 (2008).

11. Zeisberg, E. M. et al. Endothelial-to-mesenchymal transition contributes to cardiac fibrosis. Nat. Med. 13, 952-961. https://doi. org/10.1038/nm1613 (2007).

12. Wentzel, J. J. et al. Endothelial shear stress in the evolution of coronary atherosclerotic plaque and vascular remodelling: Current understanding and remaining questions. Cardiovasc. Res. 96, 234-243. https://doi.org/10.1093/cvr/cvs217 (2012).

13. Texon, M. A hemodynamic concept of atherosclerosis, with particular reference to coronary occlusion. AMA Arch. Intern. Med. 99, 418-427. https://doi.org/10.1001/archinte.1957.00260030100010 (1957).

14. Kim, M. et al. Laminar flow activation of ERK5 protein in vascular endothelium leads to atheroprotective effect via NF-E2-related factor 2 (Nrf2) activation. J. Biol. Chem. 287, 40722-40731. https://doi.org/10.1074/jbc.M112.381509 (2012).

15. Le, N. T. et al. Identification of activators of ERK5 transcriptional activity by high-throughput screening and the role of endothelial ERK5 in vasoprotective effects induced by statins and antimalarial agents. J. Immunol. 193, 3803-3815. https://doi.org/10.4049/ jimmunol.1400571 (2014).

16. Le, N. T. et al. A crucial role for p90RSK-mediated reduction of ERK5 transcriptional activity in endothelial dysfunction and atherosclerosis. Circulation 127, 486-499. https://doi.org/10.1161/CIRCULATIONAHA.112.116988 (2013).

17. Kumar, A. et al. Histone and DNA methylation-mediated epigenetic downregulation of endothelial Kruppel-like factor 2 by lowdensity lipoprotein cholesterol. Arterioscler. Thromb. Vasc. Biol. 33, 1936-1942. https://doi.org/10.1161/ATVBAHA.113.301765 (2013).

18. Maleszewska, M., Vanchin, B., Harmsen, M. C. \& Krenning, G. The decrease in histone methyltransferase EZH2 in response to fluid shear stress alters endothelial gene expression and promotes quiescence. Angiogenesis 19, 9-24. https://doi.org/10.1007/ s10456-015-9485-2 (2016).

19. Dreger, H. et al. Epigenetic regulation of cell adhesion and communication by enhancer of zeste homolog 2 in human endothelial cells. Hypertension 60, 1176-1183. https://doi.org/10.1161/HYPERTENSIONAHA.112.191098 (2012).

20. Greissel, A. et al. Histone acetylation and methylation significantly change with severity of atherosclerosis in human carotid plaques. Cardiovasc. Pathol. 25, 79-86. https://doi.org/10.1016/j.carpath.2015.11.001 (2016). 
21. Kondoh, K. \& Nishida, E. Regulation of MAP kinases by MAP kinase phosphatases. Biochim. Biophys. Acta 1773, 1227-1237. https://doi.org/10.1016/j.bbamcr.2006.12.002 (2007).

22. Smits, M. et al. Down-regulation of miR-101 in endothelial cells promotes blood vessel formation through reduced repression of EZH2. PLoS ONE 6, e16282. https://doi.org/10.1371/journal.pone.0016282 (2011).

23. Pawlyn, C. et al. Overexpression of EZH2 in multiple myeloma is associated with poor prognosis and dysregulation of cell cycle control. Blood Cancer J. 7, e549. https://doi.org/10.1038/bcj.2017.27 (2017).

24. Zhang, H. et al. Oncogenic deregulation of EZH2 as an opportunity for targeted therapy in lung cancer. Cancer Discov. 6, 10061021. https://doi.org/10.1158/2159-8290.CD-16-0164 (2016).

25. Agarwal, V., Bell, G. W., Nam, J. W. \& Bartel, D. P. Predicting effective microRNA target sites in mammalian mRNAs. Elife 4, e05005. https://doi.org/10.7554/eLife.05005 (2015).

26. Chen, P. Y. et al. FGF regulates TGF-beta signaling and endothelial-to-mesenchymal transition via control of let-7 miRNA expression. Cell Rep. 2, 1684-1696. https://doi.org/10.1016/j.celrep.2012.10.021 (2012).

27. Kovacic, J. C. et al. Endothelial to mesenchymal transition in cardiovascular disease: JACC state-of-the-art review. J. Am. Coll. Cardiol. 73, 190-209. https://doi.org/10.1016/j.jacc.2018.09.089 (2019).

28. Vanchin, B. et al. MicroRNA-374b induces endothelial-to-mesenchymal transition and early lesion formation through the inhibition of MAPK7 signaling. J. Pathol. 247, 456-470. https://doi.org/10.1002/path.5204 (2019).

29. Boon, R. A. et al. KLF2 suppresses TGF-beta signaling in endothelium through induction of Smad7 and inhibition of AP-1. Arterioscler. Thromb. Vasc. Biol. 27, 532-539. https://doi.org/10.1161/01.ATV.0000256466.65450.ce (2007).

30. Lee, E. S., Boldo, L. S., Fernandez, B. O., Feelisch, M. \& Harmsen, M. C. Suppression of TAK1 pathway by shear stress counteracts the inflammatory endothelial cell phenotype induced by oxidative stress and TGF-beta1. Sci. Rep. 7, 42487. https://doi.org/10. 1038/srep42487 (2017).

31. Moonen, J. R. et al. Endothelial progenitor cells give rise to pro-angiogenic smooth muscle-like progeny. Cardiovasc. Res. 86, 506-515. https://doi.org/10.1093/cvr/cvq012 (2010).

32. Adam, P. J., Regan, C. P., Hautmann, M. B. \& Owens, G. K. Positive- and negative-acting Kruppel-like transcription factors bind a transforming growth factor beta control element required for expression of the smooth muscle cell differentiation marker SM22alpha in vivo. J. Biol. Chem. 275, 37798-37806. https://doi.org/10.1074/jbc.M006323200 (2000).

33. Reddy, S. T. et al. Potential role for mitogen-activated protein kinase phosphatase-1 in the development of atherosclerotic lesions in mouse models. Arterioscler. Thromb. Vasc. Biol. 24, 1676-1681. https://doi.org/10.1161/01.ATV.0000138342.94314.64 (2004).

34. Shen, J. et al. Lack of mitogen-activated protein kinase phosphatase-1 protects ApoE-null mice against atherosclerosis. Circ. Res. 106, 902-910. https://doi.org/10.1161/CIRCRESAHA.109.198069 (2010).

35. Drew, B. A., Burow, M. E. \& Beckman, B. S. MEK5/ERK5 pathway: The first fifteen years. Biochim. Biophys. Acta 37-48, 2012. https://doi.org/10.1016/j.bbcan.2011.10.002 (1825).

36. Brondello, J. M., Pouyssegur, J. \& McKenzie, F. R. Reduced MAP kinase phosphatase-1 degradation after p42/p44MAPK-dependent phosphorylation. Science 286, 2514-2517. https://doi.org/10.1126/science.286.5449.2514 (1999).

37. Cao, W., Bao, C., Padalko, E. \& Lowenstein, C. J. Acetylation of mitogen-activated protein kinase phosphatase-1 inhibits toll-like receptor signaling. J. Exp. Med. 205, 1491-1503. https://doi.org/10.1084/jem.20071728 (2008).

38. Jeong, Y. et al. Histone deacetylase isoforms regulate innate immune responses by deacetylating mitogen-activated protein kinase phosphatase-1. J. Leukoc. Biol. 95, 651-659. https://doi.org/10.1189/jlb.1013565 (2014).

39. Liu, R. M. et al. Oxidative modification of nuclear mitogen-activated protein kinase phosphatase 1 is involved in transforming growth factor beta1-induced expression of plasminogen activator inhibitor 1 in fibroblasts. J. Biol. Chem. 285, 16239-16247. https:// doi.org/10.1074/jbc.M110.111732 (2010).

40. Tephly, L. A. \& Carter, A. B. Differential expression and oxidation of MKP-1 modulates TNF-alpha gene expression. Am. J. Respir. Cell Mol. Biol. 37, 366-374. https://doi.org/10.1165/rcmb.2006-0268OC (2007).

41. Jeffrey, K. L., Camps, M., Rommel, C. \& Mackay, C. R. Targeting dual-specificity phosphatases: Manipulating MAP kinase signalling and immune responses. Nat. Rev. Drug Discov. 6, 391-403. https://doi.org/10.1038/nrd2289 (2007).

42. Molina, G. et al. Zebrafish chemical screening reveals an inhibitor of Dusp6 that expands cardiac cell lineages. Nat. Chem. Biol. 5 , 680-687. https://doi.org/10.1038/nchembio.190 (2009).

43. Feng, B. et al. miR-200b mediates endothelial-to-mesenchymal transition in diabetic cardiomyopathy. Diabetes 65, 768-779. https:// doi.org/10.2337/db15-1033 (2016).

44. Wilson, P. W. Established risk factors and coronary artery disease: The Framingham study. Am. J. Hypertens. 7, 7S-12S. https:// doi.org/10.1093/ajh/7.7.7s (1994).

45. Price, J. F. et al. Relationship between smoking and cardiovascular risk factors in the development of peripheral arterial disease and coronary artery disease: Edinburgh artery study. Eur. Heart J. 20, 344-353. https://doi.org/10.1053/euhj.1998.1194 (1999).

46. Watkins, H. \& Farrall, M. Genetic susceptibility to coronary artery disease: From promise to progress. Nat. Rev. Genet. 7, 163-173. https://doi.org/10.1038/nrg1805 (2006).

47. Dichgans, M. et al. Shared genetic susceptibility to ischemic stroke and coronary artery disease: A genome-wide analysis of common variants. Stroke 45, 24-36. https://doi.org/10.1161/STROKEAHA.113.002707 (2014).

48. Zhou, T. et al. Mutant MAPK7-induced idiopathic scoliosis is linked to impaired osteogenesis. Cell Physiol. Biochem. 48, 880-890. https://doi.org/10.1159/000491956 (2018).

49. Tesser-Gamba, F. et al. MAPK7 variants related to prognosis and chemotherapy response in osteosarcoma. Ann. Diagn. Pathol. 46, 151482. https://doi.org/10.1016/j.anndiagpath.2020.151482 (2020).

50. Qiu, F. et al. A functional polymorphism in the promoter of ERK5 gene interacts with tobacco smoking to increase the risk of lung cancer in Chinese populations. Mutagenesis 28, 561-567. https://doi.org/10.1093/mutage/get033 (2013).

51. Ling, Z. et al. Effects of four single nucleotide polymorphisms of EZH2 on cancer risk: A systematic review and meta-analysis. Onco Targets Ther. 11, 851-865. https://doi.org/10.2147/OTT.S158173 (2018).

52. Yoon, K. A., Gil, H. J., Han, J., Park, J. \& Lee, J. S. Genetic polymorphisms in the polycomb group gene EZH2 and the risk of lung cancer. J. Thorac. Oncol. 5, 10-16. https://doi.org/10.1097/JTO.0b013e3181c422d9 (2010).

53. Paolicchi, E., Fornaro, L., Landi, S., Rigas, S. \& Crea, F. EZH2 single nucleotide variants (SNVs): Diagnostic and prognostic role in 10 solid tumor types. Epigenomes 1, 18. https://doi.org/10.3390/epigenomes1030018 (2017).

54. Wei-wei, T. et al. Effects of EZH2 polymorphisms on susceptibility to various carcinomas: Evidence from 6 publications. Chemotherapy 7, 1-7. https://doi.org/10.4172/2167-7700.1000252 (2018).

55. Orekhov, A. N., Andreeva, E. R., Andrianova, I. V. \& Bobryshev, Y. V. Peculiarities of cell composition and cell proliferation in different type atherosclerotic lesions in carotid and coronary arteries. Atherosclerosis 212, 436-443. https://doi.org/10.1016/j.ather osclerosis.2010.07.009 (2010).

56. Stary, H. C. et al. A definition of initial, fatty streak, and intermediate lesions of atherosclerosis. A report from the Committee on Vascular Lesions of the Council on Arteriosclerosis. Am. Heart Assoc. Circ. 89, 2462-2478. https://doi.org/10.1161/01.cir.89.5. 2462 (1994).

57. Aird, W. C. Endothelial cell heterogeneity. Crit. Care Med. 31, S221-230. https://doi.org/10.1097/01.CCM.0000057847.32590.C1 (2003). 
58. Burridge, K. A. \& Friedman, M. H. Environment and vascular bed origin influence differences in endothelial transcriptional profiles of coronary and iliac arteries. Am. J. Physiol. Heart Circ. Physiol. 299, H837-846. https://doi.org/10.1152/ajpheart.00002. 2010 (2010).

59. Lacorre, D. A. et al. Plasticity of endothelial cells: Rapid dedifferentiation of freshly isolated high endothelial venule endothelial cells outside the lymphoid tissue microenvironment. Blood 103, 4164-4172. https://doi.org/10.1182/blood-2003-10-3537 (2004).

60. Chi, J. T. et al. Endothelial cell diversity revealed by global expression profiling. Proc. Natl. Acad. Sci. U S A 100, 10623-10628. https://doi.org/10.1073/pnas.1434429100 (2003).

61. Ohura, N. et al. Global analysis of shear stress-responsive genes in vascular endothelial cells. J. Atheroscler. Thromb. 10, 304-313. https://doi.org/10.5551/jat.10.304 (2003).

62. Medina-Leyte, D. J., Dominguez-Perez, M., Mercado, I., Villarreal-Molina, M. T. \& Jacobo-Albavera, L. Use of human umbilical vein endothelial cells (HUVEC) as a model to study cardiovascular disease: A review. Appl. Sci. 10, 938. https://doi.org/10.3390/ App10030938 (2020).

63. Krenning, G., Moonen, J. R., van Luyn, M. J. \& Harmsen, M. C. Vascular smooth muscle cells for use in vascular tissue engineering obtained by endothelial-to-mesenchymal transdifferentiation (EnMT) on collagen matrices. Biomaterials 29, 3703-3711. https:// doi.org/10.1016/j.biomaterials.2008.05.034 (2008).

64. Correia, A. C., Moonen, J. R., Brinker, M. G. \& Krenning, G. FGF2 inhibits endothelial-mesenchymal transition through microRNA-20a-mediated repression of canonical TGF-beta signaling. J. Cell Sci. 129, 569-579. https://doi.org/10.1242/jcs.176248 (2016).

65. Komaravolu, R. K. et al. Erk5 inhibits endothelial migration via KLF2-dependent down-regulation of PAK1. Cardiovasc. Res. 105, 86-95. https://doi.org/10.1093/cvr/cvu236 (2015).

66. Ohnesorge, N. et al. Erk5 activation elicits a vasoprotective endothelial phenotype via induction of Kruppel-like factor 4 (KLF4). J. Biol. Chem. 285, 26199-26210. https://doi.org/10.1074/jbc.M110.103127 (2010).

\section{Acknowledgements}

BV is supported by the State Training Foundation Scholarship from the Ministry of Education and Science of Mongolia (grant \#621). MS is supported by the Dutch Kidney Foundation (grant \#15OP13), BK was supported by a FAPESP Scholarship from the São Paulo Research Foundation, Brazil (grant \#2012/11871-0). MCH is supported by a "Science without Borders"-grant from the Brazilian Federal Government (grant \#401749/2012-6). JRM is supported by the Dutch Heart Foundation (grant \#2013T116) and the Netherlands CardioVascular Research Initiative (CVON): the Dutch Heart Foundation, Dutch Federation of University Medical Centers, the Netherlands Organization for Health Research and Development, and the Royal Netherlands Academy of Sciences (CVON Phaedra 2012-08). GK is supported by innovational research incentive grants from the Netherlands Organization for Scientific Research (NWO) and the Netherlands Organization for Health Research and Development (ZonMW, grant \#916.11.022 and \#917.16.446). Imaging was performed at the UMCG Imaging Center (UMIC), supported by the Netherlands Organization for Health Research and Development (ZonMW grant \#40-00506-98-9021).

\section{Author contributions}

B.V., M.S., R.G., M.B. performed experiments and acquired the data. B.K., A.P. recruited clinical samples. M.H., J.M., G.K. designed the work. All authors were involved in interpretation of data. B.V., M.S. drafted the manuscript. G.K. made critical revisions to the manuscript. All authors have approved the submitted version and have agreed both to be personally accountable for the author's own contributions.

\section{Competing interests}

The authors declare no competing interests.

\section{Additional information}

Supplementary Information The online version contains supplementary material available at https://doi.org/ 10.1038/s41598-021-97127-4.

Correspondence and requests for materials should be addressed to G.K.

Reprints and permissions information is available at www.nature.com/reprints.

Publisher's note Springer Nature remains neutral with regard to jurisdictional claims in published maps and institutional affiliations.

Open Access This article is licensed under a Creative Commons Attribution 4.0 International License, which permits use, sharing, adaptation, distribution and reproduction in any medium or format, as long as you give appropriate credit to the original author(s) and the source, provide a link to the Creative Commons licence, and indicate if changes were made. The images or other third party material in this article are included in the article's Creative Commons licence, unless indicated otherwise in a credit line to the material. If material is not included in the article's Creative Commons licence and your intended use is not permitted by statutory regulation or exceeds the permitted use, you will need to obtain permission directly from the copyright holder. To view a copy of this licence, visit http://creativecommons.org/licenses/by/4.0/.

(C) The Author(s) 2021 